\title{
PATENT AUCTIONS AND BIDDING COALITIONS: STRUCTURING THE SALE OF CLUB GOODS
}

\author{
John Asker \\ Mariagiovanna Baccara \\ SangMok Lee \\ Working Paper 28602 \\ http://www.nber.org/papers/w28602 \\ NATIONAL BUREAU OF ECONOMIC RESEARCH \\ 1050 Massachusetts Avenue \\ Cambridge, MA 02138 \\ March 2021
}

We thank three anonymous referees and the editor Nicola Persico for helpful comments and suggestions. The views expressed herein are those of the authors and do not necessarily reflect the views of the National Bureau of Economic Research.

NBER working papers are circulated for discussion and comment purposes. They have not been peer-reviewed or been subject to the review by the NBER Board of Directors that accompanies official NBER publications.

(C) 2021 by John Asker, Mariagiovanna Baccara, and SangMok Lee. All rights reserved. Short sections of text, not to exceed two paragraphs, may be quoted without explicit permission provided that full credit, including $(\odot$ notice, is given to the source. 
Patent Auctions and Bidding Coalitions: Structuring the Sale of Club Goods

John Asker, Mariagiovanna Baccara, and SangMok Lee

NBER Working Paper No. 28602

March 2021

JEL No. D44,D47,K21,L14,L24,L4,O34

\section{ABSTRACT}

Auctioneers of patents are observed to allow joint bidding by coalitions of buyers. These auctions are distinguished by the good for sale being non-rivalrous, but still excludable, in consumption \{ that is, they auctions of club goods. This affects how coalitional bidding impacts auction performance. We study the implications of coalitions of bidders on second-price (or equivalently, ascending-price) auctions. Although the formation of coalitions can benefit the seller, we show that stable coalition profiles tend to consist of excessively large coalitions, to the detriment of both auction revenue and social welfare. Limiting the permitted coalition size increases efficiency and confers benefits on the seller. Lastly, we compare the revenues generated by patent auctions and multi-license auctions, and we find that the latter are superior in a large class of environments.

John Asker

Department of Economics

University of California, Los Angeles

Bunche Hall 8363

405 Hilgard Avenue

Los Angeles, CA 90095-1477

and NBER

johnasker@econ.ucla.edu

Mariagiovanna Baccara

Olin School of Business

Washington University in St.Louis

St.Louis, MO 63130

mbaccara@wustl.edu
SangMok Lee

Washington University in St Louis

One Brookings Drive

Saint Louis, MO 63130

United States

sangmoklee@wustl.edu 


\section{Introduction}

On June 27th, 2011 an auction began in which the patent portfolio of Nortel was put up for sale as part of its bankruptcy proceedings. ${ }^{1}$ The auction used an ascending-price mechanism, and it proceeded in rounds, with each bidder being required to outbid the leading bid in each round to retain eligibility in subsequent rounds. The initial bid of $\$ 900$ million dollars from Ranger (a subsidiary of Google) was followed by over 19 rounds of bidding, culminating in a winning bid of 4.5 billion dollars on June 30th, 2011.2 When the Nortel auction began, it had five bidders: Ranger, Apple, Intel, Norpax (an affiliate of RPX Corporation, which is an membership-based aggregator including over 320 firms as of 2020), and Rockstar (a consortium of Research in Motion, EMC, Ericsson, Sony, and Microsoft). ${ }^{3}$ Importantly, both at the beginning and during the course of the auction, the seller explicitly accommodated the presence of bidding coalitions. ${ }^{4}$

This article studies the incentives for coalitions, like those observed in the Nortel auction, to form in patent auctions. It then examines the impact that bidding coalitions have on auction revenues and market efficiency. It also identifies measures that sellers may implement so as to mitigate any adverse impact that such coalitions have on the ultimate sale price. Because of their relevance in practice, as well as their analytical tractability, we study these issues in the context of second-price auctions (or equivalently, ascending-price auctions). ${ }^{5}$

Central to the analytical approach are two attributes of intellectual property (IP), and patents in particular: First, patents are 'non-rivalrous,' in the sense that they can be pro-

\footnotetext{
${ }^{1}$ This account of the Nortel auction follows the report of Ernst \& Young (2011).

${ }^{2}$ This result was described in subsequent court hearings as 'record breaking ... in the patent industry generally' (see Brickley, 2011).

${ }^{3}$ Norpax dropped out in round two. Rockstar did not submit a bid in round five, resulting in a loss of eligibility. However, Rockstar regained eligibility in the same round after joining forces with Apple, with consent of the seller. Intel dropped out in round six. At the end of round six, two eligible bidders remained - Ranger and the reconstituted Rockstar. The seller at this point gave consent to Ranger and Rockstar to enter into partnership discussions with Intel and Norpax. After round eight, Ranger and Intel entered into a bidding partnership, with Ranger as the lead partner. In subsequent rounds, competition between Ranger and Rockstar drove the price of the Nortel patent portfolio up to the final price.

${ }^{4}$ The Nortel auction is a prominent example of the distinctive presence of bidding coalitions in patent auctions. Although data on the patent market is limited (a consequence of the decentralized nature of the market), industry sources report RPX and AST (another membership-based aggregator) as the largest purchasers of patents in the U.S. in 2017 (see Richardson et al., 2018).

${ }^{5}$ Ascending-price mechanisms similar to the ones used in the Nortel auction have been used extensively across industries, as documented for exampe by Milgrom (2004) and Klemperer (2004) in the context of spectum rights auctions.
} 
ductively used by multiple firms, although at some point negative externalities among users may emerge. Second, patents are 'excludable,' because additional users can be prevented from accessing a new technology protected by a patent. These attributes make patents an example of a 'club good.' ${ }^{6}$ Hence, although we focus on the patent application, the analysis presented here applies to any other club good. ${ }^{7}$ The non-rivalrous but excludable nature of club goods colors the incentives for coalitions to form in auctions, and the costs and benefits that the seller internalizes from their presence. In particular, the non-rivalrous nature of consumption means that coalition formation can increase bidders' valuations, and therefore revenues, at least over some range of coalition sizes. The narrative of coalition formation in the Nortel patent auction is suggestive of this feature. On the other hand, coalitions tend to depress the degree of competition on the market and may have a negative effect on the seller's revenue.

We explore these economic forces with a model in which a seller owns a patent that has multiple different applications. Let the number of applications be $\bar{n}$. There is a pool of $N$ potential firms interested in buying access to the underlying technology. The timing of our game is as follows: first, firms are allowed to aggregate into coalitions. Once coalitions are formed, every coalition establishes its own value for the patent based on its members' independent and private values' realizations. Once these values are determined, the auction takes place and coalitions are allowed to participate in the auction as individual bidders. Once the auction has taken place, the winning coalition allows its members to access the underlying technology (through, say, licensing). ${ }^{8}$

The process that determines each coalition's value for the patent accounts for the presence of potential negative externalities among patent users: once the patent's applications are implemented by $\bar{n}$ firms, distributing licenses further among members generates no additional value for the coalition. ${ }^{9}$ Therefore, if the winning coalition is larger than $\bar{n}$, it does not distribute licenses to all of their members, but only to $\bar{n}$ of them.

\footnotetext{
${ }^{6}$ See Buchanan (1965).

${ }^{7}$ Our setup also applies to auctions in which a bundle of goods, rather than a single one, are sold together, and coalitions of buyers (each with a single-unit demand) are allowed to participate as individual bidders (see Avery and Hendershott, 2000). In addition, Albano, Spagnolo, and Zanza (2008) and Albano (2017) have documented and discussed the widely common presence of joint bidding in procurement auctions. An argument used to justify the practice is the fact that projects are complex, and they may require very different skills and expertises to be completed. Our analysis applies to these scenarios as well.

${ }^{8}$ The Nortel auction has nuances that we abstract from for the sake of tractability. Notably, we do not allow coalitions to form or change during the course of the auction.

${ }^{9}$ For example, if two firms commercialize the same application, some value is destroyed by market competition.
} 
We model how each coalition's value for the patent is formed in two alternative ways. In the first environment, which we name 'the limited-value case,' after the coalition formation stage, each coalition larger than $\bar{n}$ arbitrarily chooses $\bar{n}$ members who then obtain a private and independent realization for the patent's value, drawn by the same distribution. Therefore, the valuation for the patent for each coalition is the sum of the realizations of the individual private valuations of its members, up to a maximum of $\bar{n}$ realizations. In the limited-value case, any coalition (weakly) larger than $\bar{n}$ has the same value distribution, and therefore the same probability of winning the auction. In the second environment, which we name "the optimized-value case,' if a coalition is larger than $\bar{n}$, all firms in the coalition obtain a value realization for the patent, and the coalition selects the firms with the $\bar{n}$ highest realizations to distribute the patent to. Hence, the coalition's valuation for the patent is the sum of the $\bar{n}$ highest realizations. If a coalition's size is less than or equal to $\bar{n}$, the limited-value case and the optimized-value case are equivalent.

We consider the process of coalition formation, which takes place before the values realize. Foreseeing each coalition's value, as well as the equilibrium outcome of the auction, firms endogenously form coalitions. Stable coalition profiles are ones that satisfy natural equilibrium constraints in the coalition-formation stage: a coalition profile is stable if no firm has a profitable unilateral deviation in joining a different coalition, or in participating in the auction as individual bidder.

Our first main results illustrate the effect of increasing the concentration of bidders by moving one firm $i$ from a (weakly) smaller to a larger coalition, when both coalitions are strictly smaller than $\bar{n}$. Such a move has several implications on the two coalitions' expected auction outcomes: (i) it changes the value distribution of the patent of both coalitions by adding firm $i$ 's realization to the valuation of the larger coalition and subtracting firm $i$ 's realization from the valuation of the smaller one; (ii) it changes the expected price paid by either of the two coalitions conditional on winning, and (iii) it increases the probability of winning the auction for the larger coalition, and it decreases the probability of winning for the smaller coalition. First, we show that such a move always increases the expected valuation of the auction's winner, and therefore increases total welfare. Second, we show that the overall benefits generated by such move for the larger coalitions are higher than the expected costs generated for the smaller one, so that the move strictly increases the joint expected payoffs of the two coalitions in the auction. In other words, an individual firm generates strictly more 
additional coalitional value by joining a larger coalition than a smaller one. ${ }^{10}$

The latter result has several important implications. First, it suggests that when coalitions of firms are allowed to participate in patent auctions there is a strong incentive for them to aggregate in coalitions large enough to exploit all the patent's applications. In particular, stable coalition profiles never include multiple coalitions strictly smaller than $\bar{n}$. However, this tendency toward aggregation is mitigated by the presence of negative externalities: once a coalitions becomes large enough (i.e., it already includes at least $\bar{n}$ firms), the additional value generated by additional members decreases, diminishing their ability to attract more firms. The result allows us to identify a set of necessary conditions for stable coalition profiles, which depends on the interplay between $\bar{n}$ and the total number of firms on the market, $N$.

Second, it allows us to derive implications on the seller's revenue under stable coalition profiles. These implications depend on the way coalitions form their valuation for the patent. In the limited-value case, we find that, once a coalition has already expanded to $\bar{n}$ firms, the addition of new firms to that coalition always (weakly) reduces the expected revenue of the seller in the auction. This result allows us to identify upper bounds for the seller's revenue: If the number of applications $\bar{n}$ is large relative to $N$, the expected revenue for the seller is very small. In particular, the expected second highest realization between the sum of $\bar{n}$ individual valuations, and the sum of $N-\bar{n}$ ones constitutes an upper bound for the expected revenue of the seller across all stable coalition profiles. As $\bar{n}$ approaches $N$, the seller's revenue converges to zero. On the other hand, if $\bar{n}$ is smaller, there can be enough firms in the industry to aggregate into multiple coalitions of size $\bar{n}$. In this case, the upper bound we identify for the seller's revenue is more significant, and it amounts to the expected second highest realization among $\lceil N / \bar{n}\rceil$ coalitions, all of size $\bar{n}$ except at most one. These results allow us to deliver a specific recommendation to allow the seller to generate the previously identified revenue's upper bound in the limited-value case. In particular, we show that if the seller introduces a ceiling on the coalitions' size equal to $\bar{n}$ (i.e., before coalitions form the seller announces that only coalitions of size up to $\bar{n}$ are allowed to participate in the auction), there is a uniquely stable coalition profile. As it turns out, such profile achieves the upper bound for the seller's revenue previously identified, and it also maximizes the expected welfare across all the coalition profiles.

\footnotetext{
${ }^{10}$ As these results pertain to coalitions strictly smaller than $\bar{n}$, they hold both in the limited-value case and in the optimized-value one.
} 
Next, we study the seller's revenue in the optimized-value case. In this case, even coalitions that are already of size $\bar{n}$ or larger can still increase their own expected value by adding new members (as they will be able to select the $\bar{n}$ highest among a larger number of draws). On one hand, for any given coalition profile, large coalitions would bid more aggressively, hence improving the seller's revenue. On the other hand, firms have an even stronger incentive to aggregate into large coalitions, resulting in fewer coalitions and lower seller's revenue. To illustrate this tendency, we show that even in the presence of very strong negative externalities $(\bar{n}=1)$, the grand coalition is the only stable coalition. Although this outcome achieves the first best from a welfare perspective (because the patent is guaranteed to be implemented by the firms with the $\bar{n}$ highest realizations in the economy), this comes at the detriment of the seller's revenue.

In addition to selling a patent through an auction, IP owners typically have the option of retaining ownership of the patent and licensing it to multiple parties through a multiobject auction. In the last part of our analysis we compare the two auction formats from the seller's revenue perspective. The comparison between the two selling mechanisms involves the following trade-off: in a multi-license auction, each firm participates in the auction as an individual bidder, and the seller's revenue is determined by the highest realization of the new technology's value among the losers of the auction. For a relatively high number of licenses on sale, this realization can be rather low. On the other hand, in a patent's auction, bids coincide with the sums of the coalition members' realized valuations. Therefore, the winning bid is the highest sum, and the seller's revenue is the second highest sum, of randomly chosen realizations. We focus our attention on gamma-distributions and, by simulation, we find that multi-license auctions dominate patent auctions as selling mechanism across the vast majority of the parameter space we investigate. We also find that patent auctions have the best chance to perform well vis-à-vis multi-license auctions when the number of applications $\bar{n}$ is relatively large, and the distribution of the valuations is relatively right-skewed (i.e., the difference between the highest realizations and the others is relatively large). 


\section{Related Literature.}

The question of how to sell IP has been examined in a literature starting from Katz and Shapiro (1985). This line of work considers the strategic choice of selling, unilaterally exploiting, or licensing IP when the seller and buyers are competitors in the product market, and transactions typically occur in the IP market via bargaining or take-it-or-leave it offers. Our approach is different as we focus on the performance and structure of auctions as selling mechanisms for IP and other club goods.

Related theoretical work has considered the commercialization of licenses in the context of auctions with negative externalities (see Hoppe, Jehiel, and Moldovanu, 2006, Jehiel and Moldovanu, 1996, and Jehiel, Moldovanu, and Stacchetti, 1996). By contrast, we focus on the club-good nature of IP, rather than the fact that the losing participants in a patent auction may experience negative externalities on the final marketplace. In this sense, our work follows a relatively sparse literature on club goods started by Buchanan (1965), with more recent work by Deb and Razzolini (2001), Baik, Kim, and Na (2001), Norman (2004), and Fang and Norman (2010). In this literature, Loertscher and Marx (2017) study optimal pricing mechanisms for intermediaries of club goods. This article differs from this literature in allowing buyers to form coalitions which act as individual entities on the club-good marketplace.

This article is also related to different strands of the auction literature. Comparing alternative auction formats and studying the optimal auction problem from the seller's perspective is a classic question in auction theory. ${ }^{11}$ In this literature the set of bidders is typically exogenous and fixed across the different auction formats and, except for a few notable exceptions, the bidders are symmetric. Our methodological approach is fundamentally different because in this article the auction format chosen by the seller endogenously determines the set of (potentially asymmetric) bidders. We choose to focus on second-price auctions (or, equivalently, ascending-price auctions) because they are empirically relevant and analytically easier. Clearly, if we had chosen a different auction format (say, a first-price auction), a different sets of bidders would arise in the coalition-formation process. In particular, studying stable coalition profiles would require characterizing the equilibrium bidding behavior for a large range of asymmetric bidders' profiles, which is well-known to be challenging for first-price auctions. ${ }^{12}$

\footnotetext{
${ }^{11}$ See Milgrom (2004) for a comprehensive overview.

${ }^{12}$ Asymmetric auctions have been studied, among others, by Maskin and Riley (2000a and 2000b), Cantillon (2008), and, more recently, by Kirkegaard (2009, 2012). For example, Kirkegaard (2012) ranks second- and
} 
Considering coalitions of buyers in auctions draws a natural parallel to the large literature on bidding rings (see the excellent overview provided by Marshall and Marx, 2012). ${ }^{13}$ In this literature, because collusive behavior tends to hurt the seller's revenue and is deemed illegal under the antitrust laws, bidders' cartels need to be self-enforcing (see for example McAfee and McMillan, 1992). Our perspective is different, because in the case of an auction for a club good, coalition formation can benefit the seller, and need not be presumptively anticompetitive. Therefore, we completely abstract from self-enforcement issues and we study plausible patterns of coalitions when they are allowed to form.

A strand of the literature on bidding rings considers the formation of collusive agreements between two bidders. Specifically, this line of research considers variations on a game in which, after individual valuations are realized, the two bidders can try to reach an agreement to prevent one of them from bidding in an upcoming auction. Because values are realized before the negotiation, the behavior of the bidders in the negotiation can signal their private information. This information can be valuable to the rival in the event that no collusive agreement is reached. This problem has been addressed by Eso and Schummer (2004) and Toyan (2017) in the context of second-price auctions, and by Rachmilevitch (2013) and Zheng (2019a) in the context of first-price auctions. ${ }^{14}$ Our article is distinguished from this line of work in several ways, notably by considering more than two bidders, adopting a different coalition formation protocol, and by focusing on club goods, for which a coalition's valuation

first-price auctions in the case of two asymmetric bidders. In our setting, characterizing stable coalition profiles in first-price auctions would require an analysis of auction outcomes for multiple asymmetric bidders. In turn, this would yield to a comparison between first- and second-price auctions in which the (endogenous) sets of bidders for each auction format are potentially different from each other. Hence, comparing alternative patent auctions' formats would be an interesting next step, but it is clearly beyond the scope of this article.

${ }^{13}$ For more recent contributions in this literature, see Chassang and Ortner (2019) Che, Condorelli, and Kim (2018), Decarolis, Goldmanis, and Penta (2020), and Decarolis and Rovigatti (2020). In particular, Decarolis, Goldmanis, and Penta (2020) study the role of bidding intermediaries in the context of online advertisment auctions. Interestingly, they address the stability of a collusive coalition, in which the members' individual bids are coordinated by one bidding intermediary. Our set-up is different in many dimensions, primarly because the incentives to form coalitions are fudamentally affected by the public-good nature of a patent. Also, in the coalition-formation game, we allow any number of coalitions to arise endogenously.

${ }^{14}$ Other contributions include Zheng (2019b), Chen and Tauman (2006) and Kivetz and Tauman (2010). Earlier work by Brusco and Lopomo (2002) considers a setting in which bidders coordinate during the course of an ascending price auction on the allocation of multiple objects among them. Agranov and Yariv (2018) undertake closely related experiments on the formation of collusive agreements through communication prior to an auction. 
depend on the coalition's size. ${ }^{15,16}$

A related line of literature considers the question of how to optimally design a sale when buyers can collude (for example, see Laffont and Martimort, 1997 and 2000, Pavlov, 2008, and Che and Kim, 2006 and 2009). These articles model cartel formation as being facilitated by an uninformed third party that proposes a mechanism to coordinate the activity of the cartel. ${ }^{17}$ This, in turn, puts further constraints on the principal's mechanism design problem. This article differs from that line of literature by taking a more applied approach and by considering the (positive) question of how bidders form coalitions given a specific set of auction environments for club goods. Notably, our approach allows for multiple coalitions to form and compete in the auction, which the approach taken by this line of work does not allow for.

Thematically this article is perhaps closest to Cho, Jewell and Vohra (2002), who consider coalition formation among budget-constrained bidders who compete in a first-price sealed bid auction. All bidders have the same budget constraint and valuation, and these are both common knowledge among all players. The coalition-formation stage is modeled using a dynamic coalition-formation game developed in Ray and Vohra (1999). ${ }^{18}$ Although there are clear distinctions (in Cho, Jewell and Vohra, 2002, coalitions form to mitigate budget constraints, there is complete information in the auction, and the coalition-formation stage unfolds through bargaining), in both our setting and Cho, Jewell, and Vohra (2002)'s the seller may benefit from the formation of coalitions among bidders

Finally, the notion that the set of players in a strategic situation is, in itself, endogenous is present in some work on club formation (see, e.g., Ellickson, Grodal, Scotchmer, and Zame, 1999, and Wooders, Cartwright, and Selten, 2006). More recently, Baccara and Yariv (2013 and 2016) have studied the (endogenous) homogeneity of players in the context of public-good games.

\footnotetext{
${ }^{15}$ Somewhat related is Garratt, Troger and Zheng (2009) which considers an ascending auction with resale. They show that the ex-post knockout structure can arise in equilibrium in ascending auctions with resale (see Porter, 1992, and Marshall and Marx, 2012, for discussion of knockout auctions in practice). This is in contrast to the ex-ante knockout studied by Graham and Marshall (1987) and Asker (2010).

${ }^{16}$ Also, Chatterjee, Mitra, and Mukherjee (2017) consider a dynamic model of coalition formation with multiple bidders with heterogeneous and publicly known valuations.

${ }^{17}$ Graham and Marshall (1987) provide an early contribution that studies the optimal size of a bidding ring that uses an ex-ante knockout auction. Lopomo, Marshall and Marx (2005) consider the efficiency of the collusive mechanism, finding that, without pre-auction communication, budget-balanced collusive mechanism can yield inefficient outcomes in an auction.

${ }^{18}$ Ray and Vohra (2015) provide a survey of related coalition formation models applied in a variety of settings.
} 


\section{The Model}

\section{Set-Up}

A seller owns a patent on a new technology, and several ex-ante identical firms are all potential adopters. Let $\mathcal{N}$ be the set of firms on the market, and $N \equiv|\mathcal{N}|>1{ }^{19}$ The patent is made available for sale in a second-price auction, in which coalitions of firms can participate as individual bidders. If the patent is sold to a coalition of firms, the coalition can make it available to multiple members.

Each firm $i$ 's private valuation $V_{i}$ is independently drawn from the same non-degenerate distribution $F$ over $\mathbb{R}_{+} \cdot{ }^{20}$ We assume that $0 \in \operatorname{supp}(F), E\left(V_{i}\right)=v>0$, and the distribution has a log-concave density $f .{ }^{21}$ In our analysis, we denote by $V_{(n, i)}$ the $i$-th highest realization among $n$ draws from the distribution $F$, and by $v_{(n, i)}$ its expected value.

The game unfolds as follows:

(1) Firms form coalitions (see Section 2.3);

(2) Firms' valuations for the patent are realized. Coalitions determine their value for the patent according to either the limited-values or the optimized-value case (see Section $2.2)$;

(3) Coalitions participate as individual bidders in a second-price auction (or, equivalently, an ascending-price auction) to buy the patent;

(4) Some or all members of the winning coalition gain access to the patent (see Section 2.2).

Before we discuss how coalitions form and determine their own valuations for the patent, some other assumptions merit discussion. As mentioned before, the assumption that the seller uses a second-price auction (or, equivalently, an ascending-price auction) is made, in

\footnotetext{
${ }^{19}$ Throughout the article, we assume $N$ to be exogenous and therefore abstract from firms' entry decisions in the auction. If that is not the case (for example, $N$ grows in the number of applications $\bar{n}$ ), our qualitative analysis still applies, with the only difference that the results will depend also on way in which $\bar{n}$ determines $N$.

${ }^{20}$ In the Online Appendix, we present the case in which all firms' valuations are known and symmetric.

${ }^{21}$ That is, $\log f$ is concave on $\operatorname{supp}(F)$. Examples of distributions with log-concave density functions include the uniform distribution (over any positive-length interval), the exponential distribution, the normal distribution (including any truncated one over $[a, b]$ for $-\infty \leq a<b \leq \infty$ ), the gamma distribution, the beta distribution, etc.
} 
part, for tractability and because of the empirical relevance of these classes of auctions in industry applications. As it will become apparent, if coalitions of different sizes arise in the coalition-formation stage, their valuations have different distributions. A second-price auction ensures bidding their own value to be each coalition's weakly dominant strategy, allowing us to characterize the equilibrium outcome of the asymmetric auction. ${ }^{22}$ Note also that we do not allow the seller to impose a reserve price on the auction. Any reserve price would affect the coalition-formation stage, yielding a different set of bidders. This substantially complicates the problem of identifying the optimal reserve price. In Section 3.2 we address this assumption further and propose an alternative, and simpler, tool that the seller can use to increase the revenue.

In our analysis we also rule out the possibility of a secondary market for the patent. Allowing for secondary markets would require to add a third stage of the game in which, after the auction has taken place, the winner can resell the patent to another firm or coalition. This would make the coalitions' valuations for the patent a function of the ex-post market outcomes and, in turn, of the whole coalitions' profile. This possibility introduces non-trivial complications and it is left to further research. ${ }^{23}$

An assumption underlying the coalition formation stage is that all firms observe the sizes of the other coalitions. ${ }^{24}$ The assumption that valuations are realized after coalitions are formed is conducive to tractability, as it allows us to abstract away from potential strategic communication issues at the time of coalition formation. ${ }^{25}$ In addition, such assumption allows us to capture applications in which coalitions carry out joint research to acquire information about the patent. We come back to the possibility of joint research in the next section, and to alternative timing assumptions in Section 5.2.

\footnotetext{
${ }^{22}$ Equilibrium bidding behavior in first-price auction with asymmetric bidders is well-known to be significantly more difficult to fully characterize as discussed in Section 1.2 (see Maskin and Riley, 2000a and 2000b, and Cantillon, 2008).

${ }^{23}$ See Garratt, Troger, and Zheng (2009) for a related model of an ascending auction with resale in the presence of collusion.

${ }^{24}$ This assumption simplifies the analysis of the coalition formation stage, but because we consider a secondprice auction, any assumption on the information structure at the time of the auction (e.g., firms observe some other coalition's members' valuations, etc.) is irrelevant for the auction's outcome.

${ }^{25}$ Coalitions' formation with asymmetric information is known to be challenging and require numerous additional assumptions. See for example Dutta and Vohra (2005), and the related references in Section 1.
} 


\section{Coalitions' Values}

In this section we illustrate how coalitions determine their own value for the patent. In general, we assume that if too many firms in the winning coalition adopt the patent, not all of them are able to enjoy its full benefits. For tractability, we represent a diminishing marginal value of the technology with an exogenously given $\bar{n} \leq N$ (which is common knowledge among all firms), such that only up to $\bar{n}$ firms in the winning coalition can extract a positive value from the new technology. If more than $\bar{n}$ firms adopt it, any firm in excess of $\bar{n}$ obtains zero value from the adoption. We model the way in which a coalition determines its value in two alternative ways, which we term limited-value case and optimized-value case, respectively.

Definition 1 (Limited-Values Case) In each coalition, at most $\bar{n}$ randomly selected firms obtain a realization of $V$ and can adopt the patent. For a coalition of size $m$, this yields the coalition's value $W=\sum_{i=1}^{\min \{m, \bar{n}\}} V_{i}$, where, for $m>\bar{n}, i=1,2, \ldots$, denote some randomly chosen firms in the coalition. ${ }^{26}$

Definition 2 (Optimized-values Case) Every firm on the market obtains a realization of $V$, and each coalition of size $m$ selects the highest $\min \{m, \bar{n}\}$ realizations among its members to determine the value of the patent. This yields the coalition's value $W=$ $\sum_{i=1}^{\min \{m, \bar{n}\}} V_{(m, i)}$.

Notice that for coalitions (weakly) smaller than $\bar{n}$, the coalition's value is equal to the sum of all members' realizations in both the limited- and the optimized-value cases. Therefore, for coalitions smaller than $\bar{n}$ the two cases are equivalent. Also, a fundamental difference between the limited-values and optimized-value cases is that, under the former, all coalitions of any size $m \geq \bar{n}$ have the same value distribution. On the other hand, under the latter, coalitions of size $m \geq \bar{n}$ can still benefit from additional members as they will allow them to select the $\bar{n}$ highest from a larger pool of realizations.

Our externalities' structure can be also interpreted as follows. Suppose that the patent has a maximum number $\bar{n}$ of distinct applications which are unrelated to each other, and that, within each coalition, a realization of $V$ represents the realized value of one application.

\footnotetext{
${ }^{26}$ Therefore, in the limited-value case, the value distribution of any coalition of size $m$ is the convolution of $\min \{m, \bar{n}\}$ individual distributions $F$.
} 
In addition, assume that that if more than $\bar{n}$ firms adopt the technology within a coalition, cumulative profits start decreasing because of increased competition in some applications' markets. ${ }^{27}$ Along these lines, $\bar{n}$ could be interpreted as the number of adopting firms that maximizes the cumulative value of the patent for any coalition of size $m>\bar{n}$. In this case, the coalition would never find it optimal to let more than $\bar{n}$ firms adopt the technology, and our assumption (any firm in excess to $\bar{n}$ obtains zero value from the adoption) would imply no loss of generality. ${ }^{28}$

In our model, after alliances among firms are formed, firms in each coalition research the applications of the patent in order to obtain a realization of $V$. The limited-value case applies when each application can be investigated by at most one member (this could be due, for example, to substantial research costs). Hence, although larger coalitions have the breadth to explore more applications than smaller ones, they still cannot obtain more than $\bar{n}$ realizations of the patent's value. Therefore, each coalition strictly larger than $\bar{n}$ randomly selects $\bar{n}$ firms that will explore the patent's applications. After the research has been concluded, those firms learn their private valuations, and they make them public within the coalition. At that point, the coalition assesses its total value of the patent and participate as a bidder in the auction.

The optimized-value case corresponds to situations in which conducting research and obtaining value realizations is relatively cheap, so that each firm is able to determine their individual valuation for the new technology. Therefore, each coalition strictly larger than $\bar{n}$ is able to observe all the realizations obtained by its members and, upon winning the auction, to maximize the coalition's profit, it gives access to the patent to the $\bar{n}$ members associated with the highest valuations.

An assumption underlying the coalitions' value formation process is that all firms' individual realizations are public information within the coalition. This allows each coalition to determine its own value without concerns of potential misreporting on the members' side. Because we are focusing on coalitions that can regulate themselves using legal contracts, we view misreporting concerns as less severe than in illegal bidding rings, which need to be necessarily self-enforcing.

\footnotetext{
${ }^{27}$ For example, consider a new type of lens, that could be applied to produce glasses, binoculars, and telescopes. However, if more than, say, one firm in the same product market adopts the new lens, joint profits in that market decline. Therefore, we have $\bar{n}=3$.

${ }^{28}$ One could consider more general externalities' structures by allowing the adoption of the technology to yield strictly decreasing (rather than constant up to $\bar{n}$, and then equal to zero) marginal returns. This extension is discussed in Section 5 .
} 
Finally, we assume that any coalition that does not win the auction gets zero payoff regardless of the number of firms adopting the new technology (i.e., there are no negative externalities on non-adopting firms). This assumptions allows us to avoid a coalition's value to depend on the number and size of other coalitions.

\section{Coalitions and Stability}

Before values are realized and the auction takes place, firms can form coalitions. Consider $\sigma=\left(\sigma_{1}, . ., \sigma_{J}\right)$ to be a partition of the set $\mathcal{N}$, or a coalition profile. ${ }^{29}$ Within each coalition profile $\sigma$, each coalition $\sigma_{j} \in \sigma$ (which could be a set of multiple firms or an individual firm) is a bidder in the auction. Let $n_{j} \equiv\left|\sigma_{j}\right|$-that is, $n_{j}$ is the size of coalition $\sigma_{j}$. Without loss of generality, let $\sigma_{1}$ be the largest coalition within $\sigma, \sigma_{2}$ the second largest, etc., and so on-that is, $n_{1} \geq n_{2} \geq \ldots \geq n_{J}$.

For coalition $\sigma_{j}$, the total value of the patent $W_{j}$ is determined as described in Section 2.2. As two coalition profiles characterized by the same profile $\left\{n_{1}, . ., n_{J}\right\}$ are ex-ante payoffindistinguishable, we treat such coalition profiles as an equivalence class and denote the resulting collection of equivalence classes by $\Sigma$. For sake of simplicity, we refer to the elements of $\Sigma$ as coalition profiles rather than equivalence classes of coalitions profiles.

Given a coalition profile $\sigma$, the payoff of coalition $\sigma_{j}$ from winning the auction is the difference between the realization of $W_{j}$ and the second highest bid. We denote the coalition's profit from an ex-ante perspective (i.e., expected at the time of the coalition formation, before private valuations realize) by $\pi\left(\sigma_{j} ; \sigma\right)$. For any coalition profile $\sigma=\left(\sigma_{1}, \sigma_{2}, \ldots, \sigma_{J}\right), \sigma_{j}$ 's expected payoff is

$$
\pi\left(\sigma_{j} ; \sigma\right)=\operatorname{Pr}\left\{\sigma_{j} \text { wins }\right\} \times \mathbf{E}\left[W_{j}-P_{j} \mid \sigma_{j} \text { wins }\right]
$$

where $P_{j}$ is the price paid by $\sigma_{j}$ conditional on winning. For standard arguments, it is immediate to see that in the second-price auction each coalition has a weakly dominant strategy in bidding their own value. In the rest of the article, we focus on equilibria of the auction stage in which bidders bid their own value. Therefore, $\pi\left(\sigma_{j} ; \sigma\right)$ is well-defined for any $\sigma$ and $\sigma_{j} \in \sigma .^{30}$

\footnotetext{
${ }^{29}$ That is, (i) for each $j, \emptyset \neq \sigma_{j} \subseteq \mathcal{N}$, (ii) for each $j \neq k, \sigma_{j} \cap \sigma_{k}=\emptyset$, and (iii) $\bigcup_{j=1}^{J} \sigma_{j}=\mathcal{N}$. Note that we do not allow the same firm to join two coalitions.

${ }^{30}$ Any tie-breaking rule we specify for the auction is inconsequential in determining the payoffs as such a rule is applied only in events in which the payoffs of the winning coalitions are zero.
} 
The following notation will be useful for our analysis. Any given set of bidders $\sigma=$ $\left(\sigma_{1}, . ., \sigma_{J}\right)$ is associated with a valuation vector $\left(W_{1}, \ldots, W_{J}\right)$. The winner of the patent is the coalition associated with the highest realization in $\left(W_{1}, \ldots, W_{J}\right)$, and the revenue raised corresponds to the second-highest realization. Denoting as $W_{(\sigma, m)}$ the $m$-th highest realization associated to the vector $\left(W_{1}, \ldots, W_{J}\right)$, and as $w_{(\sigma, m)}$ its expected value, the expected revenue of the seller given a set of bidders $\sigma$ is $R(\sigma) \equiv w_{(\sigma, 2)} \cdot{ }^{31}$

Next, we describe the equilibrium notion we adopt. As, in our model, utility is transferable, we focus on coalition profiles that satisfy the stability notion illustrated in the following two definitions.

Definition 3 (Profitable Deviation) Consider a coalition profile $\sigma=\left(\sigma_{1}, . ., \sigma_{J}\right)$. A firm $i$ in coalition $\sigma_{j} \in \sigma$ has a profitable deviation if at least one of the following is true:

1. The coalition profile $\sigma^{\prime}=\left(\sigma_{1}^{\prime}, \ldots, \sigma_{J+1}^{\prime}\right)$ with $\sigma_{j}^{\prime}=\sigma_{j} \backslash\{i\}, \sigma_{J+1}^{\prime}=\{i\}$, and $\sigma_{k}^{\prime}=\sigma_{k}$ for $k \neq j, J+1$ is such that

$$
\pi\left(\sigma_{j} ; \sigma\right)<\pi\left(\sigma_{j}^{\prime} ; \sigma^{\prime}\right)+\pi\left(\{i\} ; \sigma^{\prime}\right)
$$

2. For some $k \neq j$, the coalition profile $\sigma^{\prime}=\left(\sigma_{1}^{\prime}, \ldots, \sigma_{J}^{\prime}\right)$ with $\sigma_{j}^{\prime}=\sigma_{j} \backslash\{i\}, \sigma_{k}^{\prime}=\sigma_{k} \cup\{i\}$, and $\sigma_{h}^{\prime}=\sigma_{h}$ for $h \neq j, k$ is such that

$$
\pi\left(\sigma_{j} ; \sigma\right)+\pi\left(\sigma_{k} ; \sigma\right)<\pi\left(\sigma_{j}^{\prime} ; \sigma^{\prime}\right)+\pi\left(\sigma_{k}^{\prime} ; \sigma^{\prime}\right) .
$$

Definition 4 (Stable Coalition Profile) A coalition profile $\sigma$ is stable if no firm has a profitable deviation.

In words, the two requirements for a stable coalition profile read as follows: (1) there exist no coalition $\sigma_{j}$ and firm $i \in \sigma_{j}$ that would receive a strictly higher payoff as a singleton than the amount that her current coalition $\sigma_{j}$ is willing to pay to make her stay, and (2) there exist no transfer that a coalition $\sigma_{k}$ would be willing to pay a firm $i$ belonging to a

\footnotetext{
${ }^{31}$ Again, $R(\sigma)$ is well-defined because we focus on equilibria involving weakly dominant strategies in the auction stage.
} 
different coalition $\sigma_{j}$ that is strictly higher than the amount that coalition $\sigma_{j}$ is willing to pay to make firm $i$ stay. Each firm, taking as given how other firms aggregate, and foreseeing the equilibrium played in the auction stage, unilaterally selects to be in the coalition where her own marginal value is the highest. Stability is, therefore, a natural equilibrium condition for the coalition-formation stage. ${ }^{32}$ In what follows, for any given $N$ and $\bar{n}(\leq N)$, we denote the set of stable coalition profiles by $\Sigma^{*}(N, \bar{n})$.

The idea of a stable coalition profile is reminiscent of the notion of the core, which also requires a type of group stability. ${ }^{33}$ Nonetheless, there are some important distinctions. First, the setup is different - cooperative games normally specify exogenous group values, rather than group values that are derived endogenously from a strategic interaction (specifically, an auction). Second, under a transferable utility assumption, the core coincides with the coalition profile that maximizes the sum of all coalitions' expected payoffs. In our game, however, each coalition's expected payoff in the auction depends on the entire coalition profile. ${ }^{34} \mathrm{~A}$ deviation which is jointly profitable for the coalitions directly involved in it may impose negative externalities on other coalitions' profits. Therefore, a coalition profile that maximizes the sum of all coalitions' expected payoffs may not be stable. ${ }^{35}$ Third, we take a unilateraldeviation approach in our stability notion, whereas cooperative solutions such as the core allow for arbitrary group deviations. As the auction stage of our game is non-cooperative, we adopt this notion of stability rather than a cooperative one to make the equilibrium notions in the game internally coherent. ${ }^{36}$ In Section 5.1 , we discuss the implications of considering joint deviations on our results.

\footnotetext{
${ }^{32}$ As we take a unilateral-deviation approach throughout the article, we are agnostic with respect to the question of whether there is enough surplus within a coalition to compensate multiple members when they consider deviating simultaneously to other coalitions.

${ }^{33}$ For a recent survey on group formation and stability concepts, see Ray and Vohra (2015). See also additional references in Section 1.

${ }^{34}$ As it becomes apparent below, the entire coalition profile affects the probability of a coalition winning the auction as well as the expected price paid conditional on winning.

${ }^{35}$ More formally, for each coalition profile $\sigma=\left(\sigma_{1}, \ldots, \sigma_{J}\right)$, let the expected total welfare be $\Pi(\sigma) \equiv$ $\sum_{j=1}^{J} \pi\left(\sigma_{j} ; \sigma\right)$. A coalition profile $\sigma^{*} \in \arg \max _{\sigma \in \Sigma} \Pi(\sigma)$ may not be stable. In fact, a firm's unilateral deviation could increase the sum of the payoffs of the coalitions involved in (1) or (2), although at the same time decreasing the total welfare by lowering the payoffs of coalitions not directly involved in the deviation.

${ }^{36}$ Similar notions of stability with respect to unilateral (or bilateral) deviations are common in the matching and network literature.
} 


\section{Welfare}

In this section we discuss our model from a welfare perspective. The ex-post first best is achieved if the patent is implemented by the firms with the highest $\bar{n}$ realizations in the economy. In general, the presence of multiple coalitions of firms constitutes an impediment for this to happen: because the auction's mechanism selects the winner according to the highest sum of realizations within each coalition, some of the $\bar{n}$ highest realizations in the market could be wasted from a ex-post welfare perspective because they happen to occur in a losing coalition.

Still, from an ex-ante perspective, we can identify the coalition profile that maximizes the expected social value generated by the patent. Specifically, recall that, for any coalition profile $\sigma=\left\{\sigma_{1}, . ., \sigma_{J}\right\}$ associated with a valuation vector $\left(W_{1}, \ldots, W_{J}\right)$, we denote as $W_{(\sigma, 1)}$ the highest realization, and as $w_{(\sigma, 1)}$ its expected value. Because the winner of the auction is the coalition associated with the highest realization in $\sigma$, we define as $S(\sigma) \equiv w_{(\sigma, 1)}$ the expected welfare generated by coalition profile $\sigma$. The most efficient among all coalition profiles is the one that maximizes the expected welfare. The next result illustrates the effect of concentrating firms into larger coalitions on welfare. All proofs are in Section 6.2.

Proposition 1 (Effect of Concentration on Welfare) Let $\sigma=\left(\sigma_{1}, \ldots, \sigma_{J}\right)$ be a coalition profile such that $0<n_{j} \leq n_{k}<\bar{n}$. For any firm $i \in \sigma_{j}$, let $\sigma^{\prime}=\left(\sigma_{1}^{\prime}, \ldots, \sigma_{J}^{\prime}\right)$ be such that $\sigma_{j}^{\prime}=\sigma_{j} \backslash\{i\}, \sigma_{k}^{\prime}=\sigma_{k} \cup\{i\}$, and $\sigma_{h}^{\prime}=\sigma_{h}$ for $h \neq j, k$. Then, $S\left(\sigma^{\prime}\right)>S(\sigma)$.

Proposition 1 guarantees that, if two coalitions are strictly smaller than $\bar{n}$, and we move one firm from the (weakly) smaller to the larger coalition (therefore, increasing the concentration of firms into larger coalitions), the expected welfare strictly increases. To understand the intuition of Proposition 1, consider moving a firm $i$ from coalition $\sigma_{j}$ to $\sigma_{k}$, when both coalitions are strictly smaller than $\bar{n}$ and, for simplicity, assume that $\sigma_{j}$ and $\sigma_{k}$ are the only existing coalitions, so one of them wins the auction for sure. This move implies that the distribution of the coalition $\sigma_{k}$ 's valuation in the auction (the convolution of $n_{k}$ identical distributions $F$ ) 'increases' (in likelihood-ratio order sense) by one additional distribution $F$, whereas the distribution of coalition $\sigma_{j}$ 's valuation in the auction 'decreases' (in likelihood-ratio order sense) by one distribution $F$. For convenience, denote $X \equiv \sum_{a \in \sigma_{j} \backslash\{i\}} V_{a}$, and $Y \equiv \sum_{a \in \sigma_{k}} V_{a}$, and note that the welfare values before and after the move are $\max \left\{X+V_{i}, Y\right\}$ and $\max \left\{X, Y+V_{i}\right\}$, 
respectively. Intuitively, adding $V_{i}$ to the value that is already more likely to be larger must contribute more to the maximum between the two variables. In fact, $\max \left\{X, Y+V_{i}\right\}$ first-order stochastically dominates $\max \left\{X+V_{i}, Y\right\}$, yielding a higher expected welfare. Proposition 1 formalizes this intuition and generalizes it to the presence of other coalitions.

We make two observations pertaining to Proposition 1. First, because the result addresses coalitions that are strictly smaller than $\bar{n}$, and for such coalitions the limited-values and optimized-value cases are equivalent, Proposition 1 holds in both cases. Second, Proposition 1 implies that any coalition profile including more than one coalition strictly smaller than $\bar{n}$ cannot be optimal from a expected-welfare perspective.

To understand the implications of Proposition 1 on the social planner's preferred coalition profile, and the interplay between ex-ante efficiency and first best, we need to distinguish between the limited-values and the optimized-value cases.

\section{Welfare in the Limited-value Case}

Let us start by identifying the coalition profile that maximizes the expected welfare value in the limited-value case. Note that any coalition profile including at least one coalition strictly larger $\bar{n}$ cannot be optimal from a welfare perspective. Indeed, if all firms in excess to $\bar{n}$ separate from their coalition to form their own new coalition, the expected highest value of the new coalition profile strictly increases. On the other hand, Proposition 1 implies that any profile including more than one coalition strictly smaller than $\bar{n}$ cannot be optimal from a welfare perspective. Therefore, the coalition profile $\tilde{\sigma}$ that includes the maximum number of coalitions of size $\bar{n}$, and aggregates all the remaining firms into one smaller coalition must be optimal from an expected welfare perspective. Formally, let $\widetilde{\sigma} \equiv\left(\sigma_{1}, . ., \sigma_{K}\right)$ with $K \equiv\lceil N / \bar{n}\rceil$, $n_{1}=\ldots=n_{K-1}=\bar{n}$ and $n_{K}=N-\bar{n}(K-1)$. The next result follows directly from Proposition 1.

Corollary 1 (Welfare Maximization in the Limited-Value Case) $\tilde{\sigma}$ uniquely maximizes the expected welfare value among all coalition profiles.

Despite being the most efficient coalition profile ex-ante, profile $\widetilde{\sigma}$ still does not guarantee to achieve the ex-post first best. As noted above, because the winning coalition in $\widetilde{\sigma}$ is the one that realizes the maximum sum of the members' realizations, the first best is not achieved unless, ex-post, all the $\bar{n}$ highest realizations happen to belong to the same coalition. 


\section{Welfare in the Optimized-value Case}

In the optimized-value case, all firms in the economy always obtain a realization, and the winner of the auction is the coalition with the highest sum of the highest $\bar{n}$ realizations within the coalition. In the grand coalition $\sigma=(\mathcal{N})$, the patent's value always corresponds to sum of the $\bar{n}$ highest realizations in the economy. Therefore, in the optimized-value case, not only does the grand coalition maximizes the expected welfare, but it also guarantees that the $\bar{n}$ highest realizations in the economy are implemented ex-post. Therefore, the grand coalition uniquely achieves the first best.

\section{Stable Coalition Profiles and Seller's Revenue}

In this section we first study the stable coalition profiles and then we derive their implications on the seller's revenue and the welfare.

\section{Stable Coalition Profiles}

We start the analysis by addressing the existence of a stable coalition profile in our setting. The next result shows that the grand coalition is stable under a mild assumption on the distribution $F$ in the limited-values model, and it is always stable in the optimized-values model.

\section{Lemma 1 (Existence of a Stable Coalition Profile)}

(a) In the limited-value case, if $3 v_{(\bar{n}+1,2)}+\sum_{h=3}^{\bar{n}+1} v_{(\bar{n}+1, h)} \geq v_{(\bar{n}+1,1)}$, then the grand coalition is stable;

(b) In the optimized-value case, the grand coalition is always stable.

Part (a) of Lemma 1 provides a sufficient condition on $F$ for the existence of a stable coalition profile in the limited-value case. This condition is satisfied for a large class of distributions $F .{ }^{37}$ Intuitively, consider a firm $i$ that may deviate from the grand coalition and

\footnotetext{
${ }^{37}$ For example, if each firm $i$ 's private valuation's distribution is $V_{i} \sim U[0,1]$, the condition in Part (a) of Lemma 1 becomes $\frac{\bar{n}+1}{\bar{n}+2} \leq 3 \frac{\bar{n}}{\bar{n}+2}+\frac{\bar{n}-1}{\bar{n}+2}+\cdots+\frac{1}{\bar{n}+2}$, which holds for every $\bar{n} \geq 1$. Also, if $V_{i} \sim \exp (\beta)$, we have $V_{(\bar{n}+1, \bar{n}+1)} \sim \exp ((\bar{n}+1) \beta)$ and $v_{(\bar{n}+1, \bar{n}+1)}=\frac{1}{\beta} \frac{1}{\bar{n}+1}$. Because the exponential distribution is memoryless, the difference between $V_{(\bar{n}+1, \bar{n})}$ and $V_{(\bar{n}+1, \bar{n}+1)}$ follows an independent exponential with parameter $\bar{n} \beta$, which results in $v_{(\bar{n}+1, \bar{n})}=\frac{1}{\beta}\left(\frac{1}{\bar{n}+1}+\frac{1}{\bar{n}}\right)$. Similarly, $v_{(\bar{n}+1, i)}=\frac{1}{\beta}\left(\frac{1}{\bar{n}+1}+\frac{1}{\bar{n}}+\cdots+\frac{1}{i}\right)$. Therefore, the condition in Part
} 
form a coalition $\{i\}$ by itself. The singleton coalition can win the auction and realize a positive profit only in the scenario in which firm $i$ 's realization is the highest among $\bar{n}+1$ draws from the distribution $F$, and the sum of all other $\bar{n}$ draws is lower than firm $i$ 's realization. In this scenario, the price paid by coalition $\{i\}$ is the sum of the other $\bar{n}$ realizations. Therefore, the grand coalition tends to be stable if $v_{(\bar{n}+1,1)}$ is low relative to the sum all other order statistics $v_{(\bar{n}+1, h)}$ for $h=2, \ldots, \bar{n}+1$. Part (b) of Lemma 1 guarantees that, in the optimized-values model, the grand coalition is always stable. This follows immediately from the fact that the grand coalition always achieves the maximum feasible payoff in this market, i.e., the sum of the $\bar{n}$ highest realizations across all $N$ firms.

Next, in Proposition 2, we describe an important and general property of the coalitions' auction equilibrium payoffs. In the proof (which is in Section 6.2), we exploit some tools drawn from the literature on stochastic orders. For ease of reference, these tools are summarized in Section 6.1.

Proposition 2 (Effect of Concentration on Coalitions' Payoffs) In both the limited-values and optimized-value cases, let $\sigma=\left(\sigma_{1}, \ldots, \sigma_{J}\right)$ be a coalition profile such that $0<$ $n_{j} \leq n_{k}<\bar{n}$. For any firm $i \in \sigma_{j}$, let $\sigma^{\prime}=\left(\sigma_{1}^{\prime}, \ldots, \sigma_{J}^{\prime}\right)$ be such that $\sigma_{j}^{\prime}=\sigma_{j} \backslash\{i\}$, $\sigma_{k}^{\prime}=\sigma_{k} \cup\{i\}$, and $\sigma_{h}^{\prime}=\sigma_{h}$ for $h \neq j, k$. Then,

$$
\pi\left(\sigma_{j} ; \sigma\right)+\pi\left(\sigma_{k} ; \sigma\right)<\pi\left(\sigma_{j}^{\prime} ; \sigma^{\prime}\right)+\pi\left(\sigma_{k}^{\prime} ; \sigma^{\prime}\right) .
$$

Proposition 2 explores the consequences of moving one firm from a (weakly) smaller to a larger coalition, when both initial coalitions are strictly smaller than $\bar{n}$. Our result guarantees that this move always strictly increases the two coalitions' joint payoffs.

Similarly to Proposition 1, because Proposition 2 pertains to coalitions that are strictly smaller than $\bar{n}$, and for such coalitions the limited-values and optimized-value cases coincide, Proposition 2 holds in both cases. Moreover, note that Proposition 2 holds without the additional assumption on $F$ imposed in Part (a) of Lemma 1.

To understand Proposition 2, intuitively, consider a unilateral move of a firm $i$ from coalition $\sigma_{j}$ to $\sigma_{k}$, such that $n_{j} \leq n_{k}<\bar{n}$. Such move has several implications on the expected (a) of Lemma 1 holds for every $\bar{n} \geq 1$ because $2 v_{(\bar{n}+1,2)}+\left(v_{(\bar{n}+1,2)}-v_{(\bar{n}+1,1)}\right)=\frac{1}{\beta}\left[2\left(\frac{1}{\bar{n}+1}+\cdots+\frac{1}{2}\right)-1\right]>0$. In fact, for any distribution $F$ with a bounded support, the condition is always satisfied for large enough $\bar{n}$, because the difference $v_{(\bar{n}+1,1)}-v_{(\bar{n}+1,2)}$ is strictly decreasing in $\bar{n}$. 
payoffs of coalitions $\sigma_{k}$ (now $\sigma_{k}^{\prime}$ ) and $\sigma_{j}$ (now $\sigma_{j}^{\prime}$ ). For convenience, denote $X \equiv \sum_{a \in \sigma_{j} \backslash\{i\}} V_{a}$, and $Y \equiv \sum_{a \in \sigma_{k}} V_{a}$.

First, (i) the move of firm $i$ from coalition $\sigma_{j}$ to $\sigma_{k}$ increases the two coalitions' total expected valuation, conditional on the event that either of them wins. This effect is the same that underlies Proposition 1, so the same intuition we discussed in Section 2.4 applies.

Second, (ii) the move of firm $i$ from coalition $\sigma_{j}$ to $\sigma_{k}$ decreases the price paid by the winning coalition, conditional on the event that either of them wins. Again, for simplicity assume that $\sigma_{j}$ and $\sigma_{k}$ are the only two coalitions on the market, so one of them wins for sure, and the price is the realized valuation of the other coalition. Observe that the prices paid by the winner before and after the move are $\min \left\{X+V_{i}, Y\right\}$ and $\min \left\{X, Y+V_{i}\right\}$, respectively. Along the same lines as before, adding the realization of $V_{i}$ to the coalition that is less likely to have the lower realization tends to reduce the minimum between the two values. In particular, $\min \left\{X+V_{i}, Y\right\}$ first-order stochastically dominates $\min \left\{X, Y+V_{i}\right\}$.

Third (iii), in the presence of other coalitions on the market, the move of firm $i$ from coalition $\sigma_{j}$ to $\sigma_{k}$ increases the probability of the event that either of the two coalitions wins. This is because, as we already observed, $\max \left\{X, Y+V_{i}\right\}$ first-order stochastically dominates $\max \left\{X+V_{i}, Y\right\}$. Therefore, $\max \left\{X, Y+V_{i}\right\}$ has a better chance to generate the highest realization on the market when other bidders are present.

Therefore, the move of firm $i$ from coalition $\sigma_{j}$ to coalition $\sigma_{k}$ increases the two coalitions' total expected valuation and decreases the price paid by the winning coalition conditional on either of them winning the auction (effects (i) and (ii)). The move also increases the probability of the event that either of the two firms wins (effect (iii)). Hence, it must be the case that the move increases the joint expected payoffs of the two coalitions in the auction, as Proposition 2 indeed guarantees. ${ }^{38,39}$

It is interesting to compare Proposition 1 and Proposition 2. In particular, Proposition 1 addresses coalition profiles' efficiency and it guarantees that by moving firm $i$ from coalition $\sigma_{j}$ to $\sigma_{k}$ the winner's expected valuation for the patent (i.e., the expected welfare) always increases. This force corresponds solely to effect (i) listed above. When all coalitions (except

\footnotetext{
${ }^{38}$ Moreover, even if it is not relevant to our argument, the move for firm $i$ from $\sigma_{j}$ to $\sigma_{k}$ changes the probabilities of winning the auction of all other coalitions $\sigma_{h} \neq \sigma_{j}, \sigma_{k}$, as well as the expected price paid conditional on any of them winning.

${ }^{39}$ The assumption $0 \in \operatorname{supp}(F)$ ensures that small coalitions can still win the auction with positive probability. Otherwise, the strict inequality in Proposition 2 may not hold as small coalitions may get zero expected payoffs regardless of how a firm may deviate from one coalition to another.
} 
at most one) reach size $\bar{n}$, further aggregation of firms into larger coalition starts being socially wasteful. This observation allows Corollary 1 to identify $\tilde{\sigma}$ as the most efficient coalition profile. On the other hand, although Proposition 2 guarantees that stable coalition profiles cannot include multiple coalitions smaller than $\bar{n}$, it stops short of guaranteeing that the most efficient profile $\tilde{\sigma}$ is stable. This is because stability involves additional effects besides ( $i$ ): specifically, once a coalition reaches size $\bar{n}$, it could still have an incentive to add additional members in a socially wasteful way to decrease the price paid in case it wins the auction, or to increase the probability of winning. In other words, effects (ii) and (iii) can still be present if coalition $\sigma_{k}$ is (weakly) larger than $\bar{n}$.

Corollaries 2 and 3 explore the implications of Proposition 2 on the set of stable coalition profiles. They illustrate some necessary conditions for any coalition profile to be stable, which we use in the next sections to study the seller's revenue.

Corollary 2 (Small Coalitions) In both the limited-values and optimized-value cases, any stable coalition profile can include at most one coalition strictly smaller than $\bar{n}$.

Corollary 3 (Stable Coalition Profiles) In both the limited-values and optimized-value cases, for any stable coalition profile $\sigma=\left(\sigma_{1}, \ldots, \sigma_{J}\right)$,

(a) if $\bar{n}=N$, then $\sigma$ is the grand coalition;

(b) if $\frac{N}{2} \leq \bar{n}<N$, then either (i) $\bar{n} \leq n_{1}<N$, and $n_{2}=N-n_{1}$; or (ii) $\sigma$ is the grand coalition;

(c) if $\bar{n}<\frac{N}{2}$, then either (i) $n_{1}, . . n_{J} \geq \bar{n}$; or (ii) $n_{1}, . . n_{J-1} \geq \bar{n}$ and $n_{J}<\bar{n}$.

By Corollary 2, a stable coalition profile cannot have more than one coalition strictly smaller than $\bar{n}$. Thus, if $\bar{n}=N$, only the grand coalition can be a stable coalition profile (Lemma 1 identifies sufficient conditions for it to be stable). If $\frac{N}{2} \leq \bar{n}<N$, there are not enough firms to form three coalitions and still satisfy Corollary 2. Therefore, a stable coalition profile can contain at most two coalitions, the (weakly) larger one must contain at least $\bar{n}$ firms, and the smaller one the remaining ones. Finally, if $\bar{n}<\frac{N}{2}$, stable coalition profiles can contain three coalitions or more, as long as all coalitions but one are weakly larger than $\bar{n}$-that is, $n_{J-1} \geq \bar{n}$. 
To conclude, given the necessary conditions for stability illustrated in Corollary 3, a natural question to ask is whether coalition profiles other than the grand coalition can be stable or uniquely stable. The following example addresses this question in the limited-value case by showing that a coalition profile with multiple coalitions can be in fact stable (the proofs of claims (a) and (b) in Example 1 are in Section 6.3). Further examples of stable coalitions profiles are presented in Section 6.1.

Example 1 (Stable Coalition Profiles) In the limited-value case, let $N=4, \bar{n}=2$, and $\tilde{\sigma}=\left(\sigma_{1}, \sigma_{2}\right)$ be such that $n_{1}=n_{2}=2$.

(a) If $V$ has an exponential distribution with parameter $\lambda>0$, then both $\widetilde{\sigma}$ and the grand coalition are stable for any $\lambda>0$.

(b) If $V$ has a Gamma distribution $(\alpha, 1)$ with $\alpha=1 / 4$, then $\widetilde{\sigma}$ is uniquely stable. ${ }^{40}$

\section{Seller's Revenue in the Limited-value Case}

Next, we focus on the limited-value case, and we address the implications of Proposition 2 on the seller's expected revenue. First, consider the effect of moving one firm from a smaller to a larger coalition on the seller's expected revenue, when both coalitions are strictly smaller than $\bar{n}$. Despite the fact that such a move increases the two coalitions' joint payoffs (as guaranteed by Proposition 2), it is easy to build examples in which the move also benefits the seller. ${ }^{41}$ Nonetheless, the next result guarantees that moving a firm from a smaller coalition toward a sizable one (i.e., larger than $\bar{n}$ ) always reduces the seller's expected revenue.

Lemma 2 (Effect of Concentration on Seller's Revenue) In the limited-value case, let $\sigma$ be a coalition profile with $\sigma_{j}, \sigma_{k} \in \sigma$ such that $0<n_{j} \leq \bar{n} \leq n_{k}$. For any firm $i \in \sigma_{j}$, let $\sigma^{\prime}=\left(\sigma_{1}^{\prime}, \ldots, \sigma_{J}^{\prime}\right)$ be such that $\sigma_{j}^{\prime}=\sigma_{j} \backslash\{i\}, \sigma_{k}^{\prime}=\sigma_{k} \cup\{i\}$, and $\sigma_{h}^{\prime}=\sigma_{h}$ for $h \neq j, k$. Then, $R(\sigma)>R\left(\sigma^{\prime}\right)$.

\footnotetext{
${ }^{40}$ As discussed in Section 4, the gamma distribution is convenient to study because the sum of $k$ random variables with a gamma distribution $(\alpha, 1)$ follows itself a gamma distribution $(k \alpha, 1)$. In fact, numerical simulations suggest that the coalition profile $\widetilde{\sigma}$ is uniquely stable for any sufficiently small $\alpha$.

${ }^{41}$ To see this, consider a scenario in which $N$ and $\bar{n}$ are both very large, and $\sigma=\left(\sigma_{1}, \sigma_{2}, \sigma_{3}\right)$, with $\sigma_{2}=\{i\}$, $\sigma_{3}=\left\{i^{\prime}\right\}$, and $\sigma_{1}=N \backslash\left\{i, i^{\prime}\right\}$. For $N$ and $\bar{n}$ large enough, $\sigma_{1}$ wins the auction almost for sure and the seller's revenue is likely to be $\max \left\{V_{i}, V_{i^{\prime}}\right\}$. Upon a deviation of $i^{\prime}$ from $\sigma_{3}$ to $\sigma_{2}$ we have $\sigma_{1}^{\prime}=\sigma_{1}, \sigma_{2}^{\prime}=\left\{i, i^{\prime}\right\}$, and the seller's revenue is likely to increase to $V_{i}+V_{i^{\prime}}$.
} 
If a firm $i$ is moved from a coalition $\sigma_{j}$ (weakly) smaller than $\bar{n}$ to a coalition $\sigma_{k}$ larger than $\bar{n}$, the value distribution of coalition $\sigma_{j}$ decreases (in a likelihood-ratio sense), but, as coalition $\sigma_{k}$ already includes $\bar{n}$ members, the value distribution of coalition $\sigma_{k}$ is not affected by the move. Therefore, firm $i$ 's contribution effectively disappears from the auction, and the seller's expected revenue strictly decreases. Hence, from the seller's perspective, profiles involving one or more coalitions strictly larger than $\bar{n}$ are dominated by coalition profiles in which any firm in excess of $\bar{n}$ is moved toward smaller coalitions, making the coalition profile more even in sizes.

Recall that for any given $N$ and $\bar{n}(\leq N)$, we denote the set of stable coalition profiles by $\Sigma^{*}(N, \bar{n})$. Also, because $W_{(\sigma, m)}$ is the $m$-th highest realization associated to the vector $\left(W_{1}, \ldots, W_{J}\right)$, and $w_{(\sigma, m)}$ is its expected value, the expected revenue of the seller given $\sigma$ is $R(\sigma) \equiv w_{(\sigma, 2)}$. Hence, the seller's maximum expected revenue from a patent auction as a function of $N$ and $\bar{n}$ is defined by

$$
R^{*}(N, \bar{n}) \equiv \max _{\sigma \in \Sigma^{*}(N, \bar{n})} R(\sigma),
$$

and $R^{*}(N, \bar{n}) \equiv 0$ if $\Sigma^{*}(N, \bar{n})=\emptyset$. Next, we exploit the necessary condition of stable coalition profiles characterized in Corollary 3 , together with Lemma 2 , to find the maximum revenue a seller can raise through a second-price (or ascending) patent auction.

Proposition 3 (Seller's Revenue in the Limited-Values Case) In the limited-value case, (i) if $\bar{n}=N, R^{*}(N, \bar{n})=0$; (ii) if $\bar{n}<N$, let $K \equiv\lceil N / \bar{n}\rceil$, and consider the coalition profile $\widetilde{\sigma} \equiv\left\{\sigma_{1}, . ., \sigma_{K}\right\}$ with $n_{1}=\ldots=n_{K-1}=\bar{n}$ and $n_{K}=N-\bar{n}(K-1)$. Then, $R^{*}(N, \bar{n}) \leq \bar{R}^{*}(N, \bar{n}) \equiv w_{(\widetilde{\sigma}, 2)}$.

If $\bar{n}=N$, point (i) of Proposition 3 is an immediate consequence of part (a) of Corollary 3: if the grand coalition is the only coalition profile that can be stable, $R^{*}(N, \bar{n})=0$. If $\bar{n}<N \leq 2 \bar{n}$, by construction we have $K=2$, and Proposition 3 is obtained observing that Lemma 2 guarantees that among all the coalition profiles described in part (b) of Corollary 3 , the profile $\widetilde{\sigma}=\left(\sigma_{1}, \sigma_{2}\right)$ in which $n_{1}=\bar{n}$ and $n_{2}=N-n_{1}$ is the one maximizing the seller's revenue. If $N \geq 2 \bar{n}+1$, Lemma 2 guarantees that, among all coalition profiles described in part (c) of Corollary 3, the one yielding the maximum potential revenue for the seller is formed by the maximum possible number of coalitions of size $\bar{n}$, and the remaining firms allocated in one 
smaller coalition. This profile corresponds to $\widetilde{\sigma}$ in Proposition 3, and the revenue generated by it is $w_{(\widetilde{\sigma}, 2)}$. Therefore, $\bar{R}^{*}(N, \bar{n}) \equiv w_{(\widetilde{\sigma}, 2)}$ represents an upper bound for the revenue that the seller can generate across all stable coalition profiles. Finally, observe that $\widetilde{\sigma}$ corresponds to the coalition profile that maximizes the expected welfare value in Corollary 1.

In the context of second-price auctions, typically sellers are able to improve their expected revenue by introducing a reserve price. In our setting, the introduction of a reserve price affects the coalition-formation stage, and therefore it is substantially more difficult to evaluate from the seller's perspective. On one hand, coalition profiles including multiple small coalitions are still not stable (it is easy to show that Proposition 2 is robust to the introduction of a reserve price). ${ }^{42}$ On the other hand, firms aggregating into larger coalitions becomes more likely to occur in stable coalition profiles. This is because unilateral deviations of firms considering participating in the auction as single bidders are less likely to be profitable (because an individual firm may face a very small probability to obtain a value realization above the reserve price). This effect tends to hurt the seller's revenue.

Although studying the implications of a reserve price as well as identifying the optimal one is beyond the scope of this article, in our setting the seller has an alternative design tool to influence the coalition-formation stage and the auction outcome, which is much simpler to evaluate. Specifically, the seller can impose bounds on coalitions' sizes that potential bidders must satisfy to be allowed to participate in the auction. In fact, Propositions 1, 2, and 3 allow us to derive a precise auction design recommendation that allows both the social planner to achieve the maximum expected welfare value, and the seller to obtain the upper bound $w_{(\widetilde{\sigma}, 2)}$ identified in Proposition 3. In Corollary 4, we show that, when $\bar{n}<N$, if, before coalitions form, the seller imposes a ceiling (i.e., a maximum) equal to $\bar{n}$ to the bidding coalitions' size, coalition profile $\widetilde{\sigma}$ becomes uniquely stable, allowing both the upper bound identified in Proposition 3 to be obtained by the seller as auction's revenue, and the expected welfare to be maximized. ${ }^{43}$

Corollary 4 (Ceiling on Coalitions' Size) In the limited-value case, let $\bar{n}<N$. If the

\footnotetext{
${ }^{42}$ To see this, observe that the proof of Proposition 2 remains unchanged if we redefine $Z^{\prime} \equiv$ $\max \left\{\max _{h \neq j, k} \sum_{a \in \sigma_{h}} V_{a}, r\right\}$, where $r$ is a reserve price.

${ }^{43}$ Although Lemma 2 guarantees that in the limited-values case setting the ceiling at $\bar{n}$ clearly dominates setting it at any higher level, setting a ceiling below $\bar{n}$ could generate even higher expected revenues for the seller. Indeed, a ceiling below $\bar{n}$ generates a larger number of smaller bidding coalitions, each with a lower value distribution (in a first-order stochastic sense). Whether this turns out to be beneficial for the seller depends on the specific distribution $F$.
} 
seller allows only coalitions of size up to $\bar{n}$ to participate in the auction, $\widetilde{\sigma}$ is uniquely stable, and it generates the revenue $\bar{R}^{*}(N, \bar{n})$.

\section{Seller's Revenue in the Optimized-value Case}

We now turn to the implications of the results in Section 3.1 on the seller's expected revenue in the optimized-value case.

The consequences of optimized values on the set of stable coalition profiles are two-fold. First, consider a firm deviating from coalition $\sigma_{j}$ to a larger coalition $\sigma_{k}$ such that $n_{j} \leq \bar{n} \leq n_{k}$. In the limited-value case, such a deviation does not change $\sigma_{k}$ 's valuation, although it decreases $\sigma_{j}$ 's valuation as well as its probability of winning the auction. As $\sigma_{j}$ is less likely to win, all other coalitions, including $\sigma_{k}$, are more likely to win (and they pay a lower price conditional on winning). On the other hand, in the optimized-value case, such a deviation does increases $\sigma_{k}$ 's valuation. In fact, if coalitions have the ability to select the highest ones among all their members' realizations, even a coalition already larger than $\bar{n}$ can still improve its own value distribution by adding more members (this is because by adding more members the coalition will be able to select the $\bar{n}$ highest realizations among a larger number of draws). Also, as $\sigma_{k}$ 's valuation increases, the chances of $\sigma_{k}$ winning the auction increase more than in the limited-value case. This implies that deviations from a coalition to another that is larger than $\bar{n}$ are more likely to be profitable than in the limited-value case. Therefore, firms have an even stronger incentive to concentrate in larger coalitions than in the limited-value case.

Second, a firm's deviation from a coalition $\sigma_{j}$ such that $n_{j} \geq \bar{n}$ to a singleton is less likely to be profitable than in the limited-value case, as the original coalition $\sigma_{j}$ 's value distribution is higher in this case. Both of these forces tend to make coalition profiles that include a small number of large coalitions relatively stable.

Similarly to the limited-value case, the incentives for firms to concentrate into large coalitions are stronger for larger $\bar{n}$. In fact, if $\bar{n}$ is small, coalitions tend to benefit less from the acquisition of additional members. The following result shows that even if $\bar{n}=1$, and therefore the incentives to aggregate in larger coalitions are minimal, in the optimized-value case, the grand coalition is uniquely stable, guaranteeing zero revenue for the seller.

Proposition 4 (Stability in the Optimized-Value Case) In the optimized-value case, assume $\bar{n}=1$. Then, the grand coalition is uniquely stable. 
To summarize the implications of the optimized-value case on the seller's revenue, observe that, because any coalition (weakly) larger than $\bar{n}$ can still improve their value distribution by adding more members, for any given coalition profile $\sigma$ the expected bids of large coalitions are going to be higher than in the limited-value case. Therefore, if several coalitions larger than $\bar{n}$ coexist in a stable coalition profile, the seller may obtain a higher revenue. However, as the firms' incentives to consolidate in large coalitions are even stronger than in the limited-value case, it is likely the case that the grand coalition becomes uniquely stable (as Proposition 4 suggests), yielding zero revenue to the seller, but, on the other hand, achieving the first-best in terms of welfare value of the patent.

As in the limited-value case, setting a ceiling on the coalitions' size at $\bar{n}$ can surely avoid the grand coalition and guarantee a positive revenue for the seller. In fact, similarly to Corollary 4 , such ceiling would make the profile $\widetilde{\sigma}$ uniquely stable, guaranteeing $w_{(\widetilde{\sigma}, 2)}$ as expected revenue for the seller. ${ }^{44}$

\section{Multi-License Auctions}

In this section we study the implications of the IP's owner auctioning off multiple licenses rather than one patent. We compare the revenue generated by a multi-license auction to the one generated by a second-price patent auction. We focus on the limited-value case as the analysis in Section 3.3 suggests that the seller's revenue tends to be lower in the optimized-value case. Therefore, multi-license auction will tend to be optimal in a greater set of environments in the optimized-value case.

If the seller opts for an auction of multiple licenses, he is able to choose the number of licenses to sell. We assume that each firm can only participate in the auction individually, because each license is going to be issued to one specific winning firm. ${ }^{45}$ Each firm $i$ 's payoff in the auction is:

\footnotetext{
${ }^{44}$ Still, in the optimized-value case this ceiling could be dominated by both lower and higher ones. To see this, consider $N=102$ and $\bar{n}=50$. Setting a ceiling at 50 results in the coalition profile $\widetilde{\sigma}=\left(\sigma_{1}, \sigma_{2}, \sigma_{3}\right)$ with $n_{1}=n_{2}=50$ and $n_{3}=2$ being uniquely stable. Still, setting a ceiling at 51 may result in a coalition profile $\sigma^{\prime}=\left(\sigma_{1}^{\prime}, \sigma_{2}^{\prime}\right)$ with $n_{1}^{\prime}=n_{2}^{\prime}=51$ being stable as well. Because in the optimized-values case the value distributions of $\sigma_{1}^{\prime}$ and $\sigma_{2}^{\prime}$ are higher (in a first-order stochastic sense) than the ones of $\sigma_{1}$ and $\sigma_{2}$, depending on the underlying distribution $F, \sigma^{\prime}$ could increase the expected revenue of the seller.

${ }^{45}$ Firms may still decide to collude illegally to manipulate the auction's price. We rule out this possibility because this article focuses on transparent agreements to share patents legally. Naturally, considering the possibility of such illicit agreements would lead the revenue generated by multiple licenses to decrease. We also assume that licenses are non-transferable.
} 


$$
\pi_{i}=\operatorname{Pr}\{i \text { wins }\} \times \mathbf{E}\left[V_{i}-P_{i} \mid i \text { wins }\right],
$$

where $P_{i}$ is the price paid by $i$ conditional on winning. The seller auctions off at most $\bar{n}$ licenses, as any additional license would be valued zero by all firms. For each choice of selling $k \leq \bar{n}$ licenses, we assume that the seller holds a $(k+1)$-th price auction, which is well-known to be revenue-equivalent to a large class of standard multi-unit auctions (see Harris and Raviv, 1981, Weber, 1983, and Maskin and Riley, 1989). ${ }^{46}$ The expected revenue from selling $k(\leq \bar{n})$ licenses is $k v_{(N, k+1)}$. Therefore, the seller's revenue from a multi-license auction is $R^{l i c} \equiv \max _{k \leq \bar{n}} k v_{(N, k+1)}$, and we have $R^{l i c} \geq \bar{n} v_{(N, \bar{n}+1)}$-that is, the expected revenue generated by selling exactly $\bar{n}$ licenses at the uniform price of the $(\bar{n}+1)$-th order statistic among $N$ draws from the distribution $F$ represents a lower bound for the revenue generated by a multi-license auction.

The comparison between the revenue raised by a patent auction and the one raised by a multi-license auction in general depends on the distribution $F$. To get some intuition, suppose that $N=K \bar{n}$ for some $K \in \mathbb{Z}_{+}$. As seen above, a lower bound for the revenue raised by a multi-license auction is identified by $\bar{n} v_{(N, \bar{n}+1)}$. The upper bound of the patent auction revenue identified in point (ii) of Proposition 3 is the seller's expected revenue when firms form $K$ coalitions of size $\bar{n}$. If the $\bar{n}$ firms with the highest ex-post realizations happen to be consolidated in one group, the seller's revenue can be at most $\sum_{i=1}^{\bar{n}} V_{(N, \bar{n}+i)}$, which is clearly below the lower bound of the multi-license auction revenue. When the firms with the ex-post highest realizations happen to be separated into different coalitions, the revenue's comparison depends on the distribution $F$. Consider for example the case $N=4$ and $\bar{n}=2$. If the seller decides to opt for a multi-license auction, the revenue is at least $2 V_{(4,3)}$. In the patent-auction case, consider two coalitions of size $\bar{n}=2$. Ex-post, the two firms with highest realizations are equally likely to be in either one coalition or separate coalitions. In the first case, the seller's revenue will be $V_{(4,3)}+V_{(4,4)}$, which is strictly lower than $2 V_{(4,3)}$. In the second case, the seller's revenue is equally likely to be either $\min \left\{V_{(4,1)}+V_{(4,4)}, V_{(4,2)}+V_{(4,3)}\right\}$ or $\min \left\{V_{(4,1)}+V_{(4,3)}, V_{(4,2)}+V_{(4,4)}\right\}=V_{(4,2)}+V_{(4,4)}$. The seller's revenue from the patent

\footnotetext{
${ }^{46}$ Conditional on bidders' i.i.d. private valuations and single-unit demands, revenue equivalence holds for any standard multi-unit auction, either sequential or sealed-bid, uniform-price or discriminatory (in the case of uniform-price either $k$-th price or $(k+1)$-th price). Note that our externalities' structure (there are no negative externalities to non-adopters) guarantees that firms have a single-unit demand for licences. Otherwise, firms may have an incentive to bid for multiple licenses to preemptively limit the adoption of the IP.
} 
auction would dominate $2 V_{(4,3)}$ when the distribution $F$ is right-skewed, so that $V_{(4,1)}$ and $V_{(4,2)}$ tend to be significantly larger than $V_{(4,3)} \cdot{ }^{47}$ In addition, the patent auction is less likely to be optimal for large $K(=N / \bar{n})$, because firms with relatively high ex-post realizations tend to be more spread out in coalitions with mid- to low- valuation firms, decreasing the second-order statistics among the coalitions' total value realizations, and therefore the seller's revenue.

These considerations suggest that (i) for a large set of parameters and distributions $F$, the seller is better off selecting a multi-license auction over a patent auction; (ii) a patent auction has the best chance to be optimal if the value distribution is relatively right-skewed, and if $K(=N / \bar{n})$ is relatively small ( $\bar{n}$ is large relative to $N)$.

To illustrate these intuitions numerically, we consider the case of valuations distributed according to a Gamma distribution-that is, $V_{i} \sim \operatorname{Gamma}(\alpha, \beta)$ for each $i \in N$. In the case of a Gamma distribution, the convolution of $\bar{n}$ random variables $V_{i}$ is another gamma distribution $\sum_{i=1}^{\bar{n}} V_{i} \sim \operatorname{Gamma}(\bar{n} \alpha, \beta) .{ }^{48}$ Therefore, the expected revenue from a patent auction in which bidders form $K$ coalitions of size $\bar{n}$, each with valuation $\sum_{i=1}^{\bar{n}} V_{i}$, can be expressed as an expectation of the second order statistics of $K$ draws from the distribution Gamma $(\bar{n} \alpha, \beta)$. To allow for comparative statics with respect to the skewness of the distribution, we choose $\beta=\sqrt{\alpha}$, hold $\operatorname{Var}\left[V_{i}\right]=\frac{\alpha}{\beta^{2}}$ fixed, and increase $\alpha$ to decrease the skewness $\frac{2}{\sqrt{\alpha}} \cdot{ }^{49}$

Figure 1 summarizes the revenue comparison for a range of different parameter values. The dots in the figure identify markets (each defined by $(\bar{n}, N=K \bar{n})$ ) in which $\bar{n} v_{(N, \bar{n}+1)}$ (the revenue generated by an auction to sell $\bar{n}$ licenses) is (weakly) larger than the expected second order statistic of $K$ draws from the distribution $\operatorname{Gamma}(\bar{n} \alpha, \beta)$ (the revenue generated by a patent auction with $K$ coalitions of size $\bar{n}$ ). The results support the intuitions (i) and

\footnotetext{
${ }^{47}$ Recall however that we are comparing a lower bound of the multi-license auction revenue and an upper bound of a patent auction: if $F$ is severely right-skewed, the seller can decide to sell fewer than $\bar{n}$ licenses in a multi-license auction, therefore increasing revenue of the multi-license auction with respect to the lower bound.

${ }^{48}$ This property makes a Gamma distribution a particularly tractable one to work with in our setting. Note that for Gamma's distributions Myerson's regularity condition holds for $\alpha \geq 1$. Therefore, for $\alpha<1$ the multi-licences auctions we are discussing are not guaranteed to be the optimal mechanism to sell multiple objects. However, they are still revenue-equivalent to any other format of multi-object auction with the same virtual surplus and empirically relevant for the purpose of our application. Maskin and Riley (1989) discuss the generality of revenue equivalence for multi-object auctions and characterize the optimal mechanism when Myerson's regularity condition is not satisfied.

${ }^{49}$ Although the expectation $E\left[V_{i}\right]=\frac{\alpha}{\beta}=\frac{\alpha}{\sqrt{\alpha}}=\sqrt{\alpha}$ is also increasing, the scale of the private valuations is irrelevant in the revenue comparison across different auction schemes.
} 
(ii) above. Observe that the revenue generated by a multi-license auction often dominates the one generated by a patent auction. When the distribution of private valuations is relatively right-skewed (i.e., $\alpha$ is smaller) the difference between the highest realizations and the other realizations is relatively large. In this scenario, a patent auction has the best chance to generate larger revenues than the multi-license auction, particularly when $K(=N / \bar{n})$ is small.

\section{Conclusion and Discussion}

In this article we study and compare alternative ways to sell IP rights or other goods that are non-rivalrous but excludable ("club goods"). If a patent is sold in a second-price (equivalently, ascending-price) auction, we analyze the potential emergence of coalitions of firms intended to participate in the auction as individual bidders. We illustrate the impact of such coalitions on the seller's revenue as well as on social welfare, we illustrate the social and private benefits of imposing a ceiling on the coalitions' sizes, and we compare the auction outcomes to the revenue generated by selling multiple licenses instead.

\section{Joint Deviations}

In our analysis we have adopted a relatively weak stability notion, which restricts the potential deviations to unilateral ones only. This stability concept is well-suited for our application: because the auction unfolds in a non-cooperative way, modeling the group-formation stage in the same way guarantees internal consistency in our game. Nonetheless, it is interesting to explore the consequences of allowing for joint deviations on our results. In the optimizedvalue case, it is possible to show that allowing for any kind of joint deviations would always yield the (efficient) grand coalition as a unique stable partition. ${ }^{50}$ In the limited-value case, allowing joint deviation would in general require conditions stronger than the ones described in part (a) of Lemma 1 for the grand coalition to be stable. That would sometimes yield environments in which the grand coalition is uniquely stable (as in the scenarios illustrated in part (a) of Example 1). On the other hand, it would still be possible to find environments in which coalition profiles other than the grand coalition are stable, and sometimes uniquely

\footnotetext{
${ }^{50}$ In particular, we allow for joint deviations in which any set of firms belonging to a coalition can form a new coalition by themselves, or migrate together to another coalition. This result is available in the Online Appendix.
} 
stable. To see it, consider the examples illustrated in part (b) of Example 1. It is easy to verify that the result is robust to the possibility of joint deviations.

\section{Further Research}

Several extensions are left to further research. It would be interesting to explore the implementation of a first-price auction, and compare both the stable coalition profiles and the seller's revenue of the two auction formats.

Next, one can consider an alternative timing in which coalitions form first, and then the seller chooses the auction mechanism based on the observed coalition profile. An exploration in this direction requires firms to forecast the optimal auction mechanism selected by the seller in the coalition-formation stage. A small literature has explored the optimal auction mechanism based on particular profiles of (asymmetric) bidders (see Bulow and Roberts, 1989, and Maskin and Riley, 2000a). In particular, Kirkegaard (2012) ranks auction formats based on the value distributions in the case of two asymmetric bidders. However, any attempt at a general characterization of the optimal mechanism runs into non-trivial complexities.

A second alternative timing option entails a setting in which all firms' valuations realize first, and then coalition formation takes place. In this model it would be possible to address questions such as whether firms with relatively high valuations tend to aggregate into the same coalition, or they tend to divide into separate coalitions. Naturally, solving this model would require to address potential strategic signaling occurring during the negotiation stage, which causes significant changes to our environment.

Finally, the range of externalities among the users of the new technology could be expanded. In our analysis, we considered the simple case in which no externalities are present if the new technology is adopted by up to $\bar{n}$ firms, and no additional value is generated if the adopters are more than $\bar{n}$. In other words, the marginal returns of the new technology are constant in the number of adopters up to $\bar{n}$, and zero afterwards. One could instead consider decreasing marginal returns in the number of adopters. In this case, the tendency of firms to form large coalitions would depend on the rate at which the marginal returns decrease. As in Proposition 2, if the rate of decrease of marginal returns is not too high, it would still be beneficial for a firm to move from a smaller coalition to a larger one. If the marginal returns decrease rapidly, that could not be the case and we should observe a larger number of small coalitions coexisting in stable coalition profiles. 


\section{Appendix}

\section{Additional Examples of Stable Coalition Profiles}

In this section we present two additional examples of coalition profiles that, similarly to Example 1 in Section 3.1, are stable and different from the grand coalition. ${ }^{51}$

Example 2 This example illustrates that in the limited-value case, if the condition in part (a) of Lemma 1 does not hold, then a coalition profile different from the grand coalition can be uniquely stable. Consider $N=2, \bar{n}=1$, and each firm's valuation is either 3 with probability $1 / 3$, or 0 otherwise. Because the grand coalition always wins the auction and makes no payment to the seller, the expected payoff from the grand coalition is $\mathbf{E}[V]=1$, i.e., the expected value of either firm's valuation. If the two firms separate into individual coalitions, we have $\sigma=\left(\sigma_{1}, \sigma_{2}\right)$ with $n_{1}=n_{2}=1$, and using the notation in the article, the sum their expected payoffs is

$$
\mathbf{E}\left[\max \left\{V_{1}, V_{2}\right\}-\min \left\{V_{1}, V_{2}\right\}\right]=\mathbf{E}\left[V_{(2,1)}-V_{(2,2)}\right]
$$

It is easy to show that $\mathbf{E}\left[V_{(2,1)}\right]=3 \cdot\left(1-(2 / 3)^{2}\right)=5 / 3$ and $\mathbf{E}\left[V_{(2,2)}\right]=3 \cdot(1 / 3)^{2}=1 / 3$. The total expected payoff from $\sigma$ dominates the grand coalition's because $4 / 3>1$. Thus, $\sigma$ is uniquely stable.

Example 3 This example illustrates that in the limited-value case, coalitions profiles other than the grand coalition may be stable along with the grand coalition, or uniquely stable. Suppose $N=4, \bar{n}=2$, and each firm's valuation $V$ has a Bernoulli distribution-that is, its values are 1 or 0 with probability $p \in(0,1)$ and $1-p$, respectively. Then, the following result holds:

Claim 1 Let $\sigma=\left(\sigma_{1}, \sigma_{2}\right)$ be such that $n_{1}=n_{2}=2$. The stable coalitions profiles are as follows:

1. (i) If $p<1-\frac{1}{\sqrt{2}}$, only $\sigma$ is stable;

(ii) If $1-\frac{1}{\sqrt{2}} \leq p \leq 1 / 2$, only $\sigma$ and the grand coalition are stable;

(iii) If $p>1 / 2$, only the grand coalition is stable.

\footnotetext{
${ }^{51}$ Note that, in a slight deviation from the article's main setup, these examples use distributions of $V$ that are not log-concave. The log-concavity assumption is used to show Proposition 2, although it is not necessary for it to hold, as Examples 2 and 3 below illustrate.
} 
Proof of Claim 1: Consider $\sigma=\left(\sigma_{1}, \sigma_{2}\right)$ such that $n_{1}=n_{2}=2$. We have,

$$
\begin{aligned}
\pi\left(\sigma_{2} ; \sigma\right) & =p^{2}\left[2(1-p)^{2}+2 p(1-p)\right]+2 p(1-p)(1-p)^{2} \\
& =2 p\left[p(1-p)^{2}+p^{2}(1-p)+(1-p)^{3}\right]
\end{aligned}
$$

which is obtained considering the scenarios in which $\sigma_{2}$ 's payoffs are strictly positive with their corresponding probabilities. For example, if $\sigma_{2}$ 's valuation is 2 , which occurs with probability $p^{2}$, then $\sigma_{2}$ 's profit (when strictly positive) can be either 2 or 1 because the valuation for $\sigma_{1}$ can be 0 or 1 with probability $(1-p)^{2}$ and $2 p(1-p)$, respectively. Therefore, we have

$$
\pi\left(\sigma_{1} ; \sigma\right)+\pi\left(\sigma_{2} ; \sigma\right)=2 \pi\left(\sigma_{2} ; \sigma\right)=4 p\left[p(1-p)^{2}+p^{2}(1-p)+(1-p)^{3}\right] .
$$

Observe that only two coalition profiles can arise from a unilateral deviation with respect to $\sigma$, and they are $\sigma^{\prime}=\left(\sigma_{1}^{\prime}, \sigma_{2}^{\prime}, \sigma_{3}^{\prime}\right)$ and $\sigma^{\prime \prime}=\left\{\sigma_{1}^{\prime \prime}, \sigma_{2}^{\prime \prime}\right\}$ such that $\left(n_{1}^{\prime}, n_{2}^{\prime}, n_{3}^{\prime}\right)=(2,1,1)$ and $\left(n_{1}^{\prime \prime}, n_{2}^{\prime \prime}\right)=(3,1)$, respectively. First, consider $\sigma^{\prime}$, obtained by a firm $i$ in $\sigma_{2}$ deviating to form $\sigma_{3}^{\prime}=\{i\}$, and observe that

$$
\pi\left(\sigma_{2}^{\prime} ; \sigma^{\prime}\right)+\pi\left(\sigma_{3}^{\prime} ; \sigma^{\prime}\right)=2 p(1-p)^{3} .
$$

This is because the only way for either $\sigma_{2}^{\prime}$ or $\sigma_{3}^{\prime}$ to obtain a strictly positive profit of 1 is for $\sigma_{1}^{\prime}$ 's valuation to be 0 , which occurs with probability $(1-p)^{2}$, and for either $\sigma_{2}^{\prime}$ or $\sigma_{3}^{\prime}$ (but not both) to have valuation 1 , which occurs with probability $2 p(1-p)$. From (3) and (4), it is easy to verify that $\pi\left(\sigma_{2} ; \sigma\right)>\pi\left(\sigma_{2}^{\prime} ; \sigma^{\prime}\right)+\pi\left(\sigma_{3}^{\prime} ; \sigma^{\prime}\right)$, implying that firm $i$ 's deviation to $\sigma^{\prime}$ is not profitable.

Second, consider $\sigma^{\prime \prime}$, obtained by a firm $i$ in $\sigma_{2}$ deviating to join $\sigma_{1}$. We have

$$
\begin{aligned}
\pi\left(\sigma_{1}^{\prime \prime} ; \sigma^{\prime \prime}\right)+\pi\left(\sigma_{2}^{\prime \prime} ; \sigma^{\prime \prime}\right) & =p^{2}(2(1-p)+p)+2 p(1-p)^{2}+p(1-p)^{2} \\
& =p^{2}(2-p)+3 p(1-p)^{2}
\end{aligned}
$$

Because $\bar{n}=2$, coalition $\sigma_{1}^{\prime \prime}$ 's valuation is 2 with probability $p^{2}$. In this scenario, $\sigma_{1}^{\prime \prime}$ can get strictly positive profits, either 2 or 1 with probabilities $1-p$ and $p$, respectively. If $\sigma_{1}^{\prime \prime}$ 's value is 1 , which occurs with probability $2 p(1-p)$, then $\sigma_{1}^{\prime \prime}$ 's profit can be at most 1 , which occurs with probability $1-p$. Finally, with probability $(1-p)^{2}, \sigma_{1}^{\prime \prime \prime}$ 's value is 0 , in which case, $\sigma_{2}^{\prime \prime}$ 
obtains a strictly positive payoff of 1 with probability $p$. From (4) and (5), we have

$$
\begin{aligned}
& \pi\left(\sigma_{1} ; \sigma\right)+\pi\left(\sigma_{2} ; \sigma\right) \geq \pi\left(\sigma_{1}^{\prime \prime} ; \sigma^{\prime \prime}\right)+\pi\left(\sigma_{2}^{\prime \prime} ; \sigma^{\prime \prime}\right) \\
& \quad \Longleftrightarrow 4 p(1-p)^{2}+4 p^{2}(1-p) \geq 2 p^{2}(1-p)+p^{3}+3 p(1-p)^{2} \\
& \quad \Longleftrightarrow(1-p)^{2}+4 p^{2}(1-p) \geq 2 p(1-p)+p^{2} \\
& \quad \Longleftrightarrow(1-2 p) \geq 2 p(1-p)-4 p^{2}(1-p)=2 p(1-p)(1-2 p) \\
& \quad \Longleftrightarrow 1-2 p \geq 0 \quad \text { (because } 2 p(1-p)<1) .
\end{aligned}
$$

Hence, $\sigma$ is stable if and only if $p \leq 1 / 2$.

Next, the expected profit of the grand coalition is

$$
\pi(\mathcal{N})=2 p^{2}+2 p(1-p)=2 p
$$

A firm $i$ 's unilaterally deviation results in the coalition profile $\sigma=\left(\sigma_{1}^{\prime \prime}, \sigma_{2}^{\prime \prime \prime}\right)$ with $n_{1}^{\prime \prime}=3$ and $n_{2}^{\prime \prime}=1$. Therefore, (5) implies

$$
\begin{aligned}
\pi(\mathcal{N}) & \geq \pi\left(\sigma_{1}^{\prime \prime} ; \sigma^{\prime \prime}\right)+\pi\left(\sigma_{2}^{\prime \prime} ; \sigma^{\prime \prime}\right) \\
& \Longleftrightarrow 2 \geq 2 p^{2}-4 p+3 \\
& \Longleftrightarrow 2(1-p)^{2} \leq 1 \\
& \Longleftrightarrow 1-\frac{1}{\sqrt{2}} \leq p .
\end{aligned}
$$

Hence, the grand coalition is stable if and only if $p \geq 1-\frac{1}{\sqrt{2}}$.

Consider now $\sigma^{\prime \prime}=\left(\sigma_{1}^{\prime \prime}, \sigma_{2}^{\prime \prime}\right)$ such that $\left(n_{1}^{\prime \prime}, n_{2}^{\prime \prime}\right)=(3,1)$. From $(6)$, a necessary condition for $\sigma^{\prime \prime}$ to be stable is $p \geq 1 / 2$. At the same time, from (7), a necessary condition for $\sigma^{\prime \prime}$ to be stable is $p \leq 1-\frac{1}{\sqrt{2}}$. As $1-\frac{1}{\sqrt{2}}<1 / 2, \sigma^{\prime \prime}$ is never stable.

Finally, as suggested by Proposition 2, it is easy to see that any profile involving two or more singleton coalitions is not stable, which concludes the proof of Claim 1.

\section{References from Shaked and Shanthikunar (2007)}

Some of the proofs presented in Section 6.2 below require some tools from the statistical literature on stochastic orders. For ease of reference, here we provide a summary of these results (see Shaked and Shanthikunar, 2007, for more detail). 
Let $X$ and $Y$ be two continuous random variables such that $P(X \geq x) \leq P(Y \geq x)$ for all $x$. Then $X$ is smaller than $Y$ in the usual stochastic order (denoted $X \leq_{s t} Y$ ). Similarly, if $X$ and $Y$ have densities $g$ and $h$, respectively, $X$ is smaller than $Y$ in likelihood-ratio order (denoted by $X \leq_{l r} Y$ ) if $g(x) h(y) \geq g(y) h(x)$ for all $x \leq y$. It is well known that likelihood-ratio dominance implies stochastic dominance. With these definitions, we can state the following theorems:

Theorem A1 (Shaked and Shanthikunar, 2007, Theorem 1.C.11) Let $X_{i}, i=1,2, \ldots$ be a sequence of non-negative independent random variables with log-concave densities. Let $M$ and $N$ be two discrete positive integer-valued random variables such that $M \leq_{l r}$ $N$, and assume that $M$ and $N$ are independent of $X_{i}, i=1,2, \ldots$ Then,

$$
\sum_{i=1}^{M} X_{i} \leq_{l r} \sum_{i=1}^{N} X_{i}
$$

Next, define $\mathcal{G}_{l r}=\left\{\phi: \mathbb{R}^{2} \rightarrow \mathbb{R}^{2}: \phi(x, y) \leq \phi(y, x)\right.$ whenever $\left.x \leq y\right\}$. Then,

Theorem A2 (Shaked and Shanthikunar, 2007, Theorem 1.C.20) Let $X$ and $Y$ be independent random variables. Then, $X \leq_{l r} Y$ if and only if

$$
\phi(X, Y) \leq_{s t} \phi(Y, X) \text { for all } \phi \in \mathcal{G}_{l r}
$$

Theorem A3 (Shaked and Shanthikunar, 2007, Theorem 1.A.3) Let $X_{1}, X_{2}, \ldots, X_{m}$ be a set of independent random variables, and let $Y_{1}, Y_{2}, \ldots, Y_{m}$ be another set of independent random variables. If $X_{i} \leq_{s t} Y_{i}$ for $i=1,2, \ldots, m$, then, for any increasing function $\psi: R^{m} \rightarrow R$, one has

$$
\psi\left(X_{1}, X_{2}, \ldots, X_{m}\right) \leq_{s t} \psi\left(Y_{1}, Y_{2}, \ldots, Y_{m}\right)
$$




\section{Proofs}

Proof of Proposition 1: Let $\sigma=\left(\sigma_{1}, \ldots, \sigma_{J}\right)$ be a coalition profile such that $0<n_{j} \leq$ $n_{k}<\bar{n}$. For any firm $i \in \sigma_{j}$, let $\sigma^{\prime}=\left(\sigma_{1}^{\prime}, \ldots, \sigma_{J}^{\prime}\right)$ be such that $\sigma_{j}^{\prime}=\sigma_{j} \backslash\{i\}, \sigma_{k}^{\prime}=\sigma_{k} \cup\{i\}$, and $\sigma_{h}^{\prime}=\sigma_{h}$ for $h \neq j, k$. In the next steps, we show that $S\left(\sigma^{\prime}\right)>S(\sigma)$, or $w_{\left(\sigma^{\prime}, 1\right)}>w_{(\sigma, 1)}$.

Step 1: We define

$$
X \equiv \sum_{a \in \sigma_{j} \backslash\{i\}} V_{a}, \quad Y \equiv \sum_{a \in \sigma_{k}} V_{a}, \quad \text { and } \quad Z \equiv \max _{h \neq j, k} \sum_{a \in \sigma_{h}} V_{a}
$$

The expected welfare values of coalitions $\sigma$ and $\sigma^{\prime}$ are

$$
w_{(\sigma, 1)}=\mathbf{E}\left[\max \left\{X+V_{i}, Y, Z\right\}\right], \quad \text { and } \quad w_{\left(\sigma^{\prime}, 1\right)}=\mathbf{E}\left[\max \left\{X, Y+V_{i}, Z\right\}\right] .
$$

We use Theorems A1 and A2 in Section 6.1. As $n_{k}>n_{j}-1$, by Theorem A1, we have $X \leq_{l r} Y$. To use Theorem A2, observe that, whenever $x \leq y, \max \left\{x+v_{i}, y, z\right\} \leq$ $\max \left\{y+v_{i}, z\right\}=\max \left\{x, y+v_{i}, z\right\}$. Hence, for every realization of $V_{i}=v_{i}$ and $Z=z$,

$$
\mathbf{E}\left[\max \left\{X+v_{i}, Y, z\right\}\right] \leq \mathbf{E}\left[\max \left\{X, Y+v_{i}, z\right\}\right]
$$

which implies

$$
\mathbf{E}\left[\max \left\{X+V_{i}, Y, Z\right\}\right] \leq \mathbf{E}\left[\max \left\{X, Y+V_{i}, Z\right\}\right]
$$

Step 2: We now find some realizations $V_{i}=v^{*}$ and $Z=0$ such that

$$
\mathbf{E}\left[\max \left\{X+v^{*}, Y, 0\right\}\right]<\mathbf{E}\left[\max \left\{X, Y+v^{*}, 0\right\}\right]
$$

Take any $v^{*}$ such that $v^{*}>0, f\left(v^{*}\right)>0$, and $\operatorname{Pr}\left(Y \geq X+v^{*}\right) \geq 1 / 2 .^{52}$ Observe that

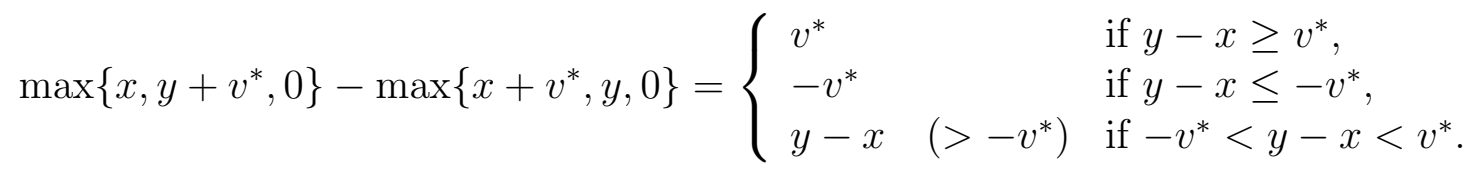

Last, $\operatorname{Pr}\left(-v^{*}<Y-X<v^{*}\right)>0$ because $0 \in \operatorname{supp}(F)$, so that the support of the distribution of $Y-X$ contains 0 . Therefore,

$\mathbf{E}\left[\max \left\{X, Y+v^{*}, 0\right\}\right]-\mathbf{E}\left[\max \left\{X+v^{*}, Y, 0\right\}\right]>v^{*}\left(\operatorname{Pr}\left(Y-X \geq v^{*}\right)-\operatorname{Pr}\left(Y-X<v^{*}\right)\right) \geq 0$.

Because the function $\max \left\{x, y+v_{i}, z\right\}-\max \left\{x+v_{i}, y, z\right\}$ is continuous in $v_{i}$ and $z$, the above strict inequality holds for every realization of $V_{i}$ and $Z$ near $v^{*}$ and 0 , respectively. Inequality

\footnotetext{
${ }^{52} \mathrm{Such} v^{*}$ exists because $Y \geq_{l r} X+V_{i}$ which implies that $\operatorname{Pr}\left(Y \geq X+V_{i}\right)=\int \operatorname{Pr}(Y \geq X+v) f(v) d v \geq 1 / 2$.
} 
(8) holds strictly because the log-concavity of $f$ guarantees that distribution functions of $V_{i}$ and $Z$ are continuous.

Proof of Lemma 1: (a) In the limited values model, consider a firm $i$, let $\sigma \equiv(\mathcal{N})$ be the grand coalition and $\sigma^{\prime} \equiv(\mathcal{N} \backslash\{i\},\{i\})$. First, consider the case in which $\bar{n}=N$. The payoff of the winning coalition of the patent auction under the profile $\sigma^{\prime}$ is $\max \left\{\sum_{a \in \mathcal{N} \backslash\{i\}} V_{a}, V_{i}\right\}-$ $V_{\left(\sigma^{\prime}, 2\right)}$. Because

$$
\max \left\{\sum_{a \in \mathcal{N} \backslash\{i\}} V_{a}, V_{i}\right\}-V_{\left(\sigma^{\prime}, 2\right)} \leq \max \left\{\sum_{a \in \mathcal{N} \backslash\{i\}} V_{a}, V_{i}\right\} \leq \sum_{a \in \mathcal{N}} V_{a}
$$

firm $i$ would not unilaterally deviate from the grand coalition.

Suppose now that $\bar{n}<N$. The payoff of the grand coalition $\sigma$ is the sum of $\bar{n}$ valuecontributing firms' private valuations. We generate the grand coalition's payoff in two steps: adding $\bar{n}+1$ draws from the distribution $F$ and excluding one of the draws with the same probability among all of them. Recall that we denote by $V_{(n, m)}$ the $m$-th highest value among $n$ draws from the distribution $F$, and that we denote by $v_{(n, m)}$ the expected value of $V_{(n, m)}$. We have

$$
\pi(\mathcal{N} ; \sigma)=\frac{1}{\bar{n}+1} \sum_{i=1}^{\bar{n}+1}\left(v_{(\bar{n}+1,1)}+v_{(\bar{n}+1,2)}+\ldots v_{(\bar{n}+1, \bar{n}+1)}-v_{(\bar{n}+1, i)}\right)
$$

Consider now the coalition profile $\sigma^{\prime}$. To obtain the coalitions' payoffs associated with $\sigma^{\prime}$, let us consider $\bar{n}+1$ draws from the distribution $F$ and select among them, with the same probability, one to be the valuation of firm $i$. Consider the case in which coalition $\{i\}$ 's realization is the highest among all draws (which happens with probability $\frac{1}{\bar{n}+1}$ ). Conditional on this case, if coalition $\{i\}$ wins the patent auction, its payoff is $V_{(\bar{n}+1,1)}-\left[V_{(\bar{n}+1,2)}+\ldots+V_{(\bar{n}+1, \bar{n})}\right]$, whereas if coalition $\mathcal{N} \backslash\{i\}$ wins the auction its payoff is $\left[V_{(\bar{n}+1,2)}+\ldots+V_{(\bar{n}+1, \bar{n})}\right]-V_{(\bar{n}+1,1)}$. Therefore, the winning coalition's expected payoff is bounded above by $\sum_{j \neq 2} V_{(\bar{n}+1, j)}-V_{(\bar{n}+1,2)}$. If coalition $\{i\}$ realizes the $h$-th highest valuation among all draws, with $h \neq 1$, coalition $\mathcal{N} \backslash\{i\}$ wins the auction and the winner's expected payoff is $\sum_{j \neq h} v_{(\bar{n}+1, j)}-v_{(\bar{n}+1, h)}$. Coalition $\{i\}$ realizes each of the $h$-th highest valuation among all draws, with $h \neq 1$ with probability $\frac{1}{\bar{n}+1}$. Thus,

$$
\pi\left(N \backslash\{i\} ; \sigma^{\prime}\right)+\pi\left(\{i\} ; \sigma^{\prime}\right)<\frac{1}{\bar{n}+1}\left[\sum_{j \neq 2} v_{(\bar{n}+1, j)}-v_{(\bar{n}+1,2)}\right]+\frac{1}{\bar{n}+1} \sum_{h=2}^{\bar{n}+1}\left[\sum_{j \neq h} v_{(\bar{n}+1, j)}-v_{(\bar{n}+1, h)}\right] .
$$


Therefore, exploiting the condition in Lemma 1's claim, we have

$$
\pi(\mathcal{N} ; \sigma)-\left[\pi\left(\mathcal{N} \backslash\{i\} ; \sigma^{\prime}\right)+\pi\left(\{i\} ; \sigma^{\prime}\right)\right]>\frac{1}{\bar{n}+1}\left(\sum_{h=3}^{\bar{n}+1} v_{(\bar{n}+1, h)}-v_{(\bar{n}+1,1)}+3 v_{(\bar{n}+1,2)}\right)>0,
$$

which guarantees that the grand coalition is stable. (b) In the optimized values model the grand coalition always achieves the maximum feasible payoff in this market, i.e., the sum of the $\bar{n}$ highest valuations across all $N$ firms on the market (i.e., $\left.\sum_{i=1}^{\bar{n}} V_{(N, i)}\right)$. Therefore, it has to be stable.

Proof of Proposition 2: Step 1: We define

$$
X \equiv \sum_{a \in \sigma_{j} \backslash\{i\}} V_{a}, \quad Y \equiv \sum_{a \in \sigma_{k}} V_{a}, \quad \text { and } \quad Z \equiv \max _{h \neq j, k} \sum_{a \in \sigma_{h}} V_{a},
$$

and observe that

$$
\begin{aligned}
& \pi\left(\sigma_{j} ; \sigma\right)+\pi\left(\sigma_{k} ; \sigma\right)=\mathbf{E}\left[\max \left\{X+V_{i}, Y, Z\right\}-\max \left\{\min \left\{X+V_{i}, Y\right\}, Z\right\}\right], \quad \text { and } \\
& \pi\left(\sigma_{j}^{\prime} ; \sigma^{\prime}\right)+\pi\left(\sigma_{k}^{\prime} ; \sigma^{\prime}\right)=\mathbf{E}\left[\max \left\{X, Y+V_{i}, Z\right\}-\max \left\{\min \left\{X, Y+V_{i}\right\}, Z\right\}\right] .
\end{aligned}
$$

Additionally, for any $x, y, v_{i}, z \geq 0$, we define

$$
\phi\left(x, y ; v_{i}, z\right) \equiv \max \left\{x+v_{i}, y, z\right\}-\max \left\{\min \left\{x+v_{i}, y\right\}, z\right\} .
$$

In the following Steps 2-3, we show that

$$
\mathbf{E}\left[\phi\left(X, Y ; V_{i}, Z\right)\right]<\mathbf{E}\left[\phi\left(Y, X ; V_{i}, Z\right)\right]
$$

which guarantees the claim of Proposition 2.

Step 2: First, we use Theorems A1 and A2 (see Section 6.1) to obtain a weak version of inequality (9). As $n_{k}>n_{j}-1$, by Theorem A1, we have $X \leq_{l r} Y$. To use Theorem A2, observe that, whenever $x \leq y$,

$$
\begin{aligned}
& \phi\left(x, y ; v_{i}, z\right)=\max \left\{x+v_{i}, y, z\right\}-\max \left\{\min \left\{x+v_{i}, y\right\}, z\right\} \quad \text { and } \\
& \phi\left(y, x ; v_{i}, z\right)=\max \left\{y+v_{i}, z\right\}-\max \{x, z\} .
\end{aligned}
$$

Hence, we have $\phi\left(x, y ; v_{i}, z\right) \leq \phi\left(y, x ; v_{i}, z\right)$. Then, for every realization of $V_{i}=v_{i}$ and $Z=z$,

$$
\mathbf{E}_{X, Y}\left[\phi\left(X, Y ; v_{i}, z\right)\right] \leq \mathbf{E}_{X, Y}\left[\phi\left(Y, X ; v_{i}, z\right)\right]
$$


which implies that

$$
\mathbf{E}\left[\phi\left(X, Y ; V_{i}, Z\right)\right] \leq \mathbf{E}\left[\phi\left(Y, X ; V_{i}, Z\right)\right]
$$

Step 3: We now find some realizations $V_{i}=v^{*}$ and $Z=0$ such that

$$
\mathbf{E}_{X, Y}\left[\phi\left(X, Y ; v^{*}, 0\right)\right]<\mathbf{E}_{X, Y}\left[\phi\left(Y, X ; v^{*}, 0\right)\right] .
$$

Take any $v^{*}$ such that $v^{*}>0, f\left(v^{*}\right)>0$, and $\operatorname{Pr}\left(Y \geq X+v^{*}\right) \geq 1 / 2 .{ }^{53}$ Then,

$$
\begin{aligned}
& \phi\left(x, y ; v^{*}, 0\right)=\max \left\{x+v^{*}, y\right\}-\min \left\{x+v^{*}, y\right\}=\left|y-x-v^{*}\right| \quad \text { and } \\
& \phi\left(y, x ; v^{*}, 0\right)=\max \left\{y+v^{*}, x\right\}-\min \left\{y+v^{*}, x\right\}=\left|y-x+v^{*}\right|
\end{aligned}
$$

Therefore,

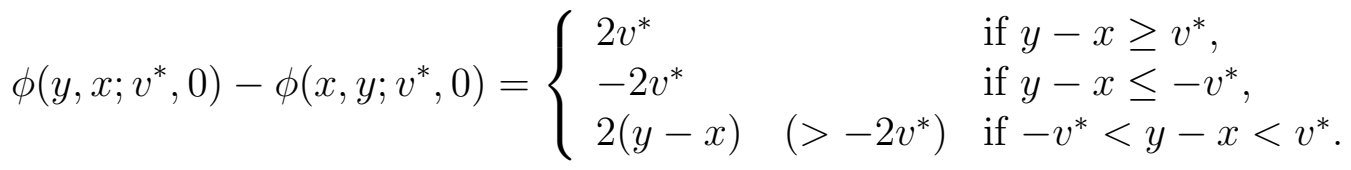

Last, $\operatorname{Pr}\left(-v^{*}<Y-X<v^{*}\right)>0$ because $0 \in \operatorname{supp}(F)$, so that the support of the distribution of $Y-X$ contains 0 . Therefore,

$$
\mathbf{E}\left[\phi\left(Y, X ; v^{*}, 0\right)-\phi\left(X, Y ; v^{*}, 0\right)\right]>2 v^{*}\left(\operatorname{Pr}\left(Y-X \geq v^{*}\right)-\operatorname{Pr}\left(Y-X<v^{*}\right)\right) \geq 0 .
$$

As $\phi$ is a continuous function of $v_{i}$ and $z$, the above strict inequality holds for every realization of $V_{i}$ and $Z$ near $v^{*}$ and 0 , respectively. Inequality (9) follows from the log-concavity of $f$, which guarantees that distribution functions of $V_{i}$ and $Z$ are continuous.

Proof of Example 1 Consider a coalition profile $\sigma=\left(\sigma_{1}, \sigma_{2}\right)$ with $n_{1}=n_{2}=2$. Observe that only two coalition profiles can arise from a unilateral deviation with respect to $\sigma$, and they are $\sigma^{\prime}=\left(\sigma_{1}^{\prime}, \sigma_{2}^{\prime}, \sigma_{3}^{\prime}\right)$ with $\left(n_{1}^{\prime}, n_{2}^{\prime}, n_{3}^{\prime}\right)=(2,1,1)$, and $\sigma^{\prime \prime}=\left\{\sigma_{1}^{\prime \prime}, \sigma_{2}^{\prime \prime}\right\}$ with $\left(n_{1}^{\prime \prime}, n_{2}^{\prime \prime}\right)=(3,1)$.

(a) Suppose that $V \sim \exp (\lambda), \lambda>0$. Recall that the condition in part (a) of Lemma 1is always satisfied for $V \sim \exp (\lambda)$ for any $\lambda \geq 0$ and $\bar{n} \geq 1$. Therefore, the grand coalition is always stable. Consider now $\sigma$, and observe that Proposition 2 guarantees that, given $\sigma$, deviating to form a new coalition $\{i\}$ is never a profitable deviation for any firm. Second, consider a firm $i$ in $\sigma_{2}$ unilaterally deviating to join $\sigma_{1}$, to obtain the new coalition profile $\sigma^{\prime \prime}$. Without the deviation, we have ${ }^{54}$

$$
\pi\left(\sigma_{1} ; \sigma\right)+\pi\left(\sigma_{2} ; \sigma\right)=\int_{0}^{\infty} \int_{0}^{\infty}|x-y|\left(\lambda^{2} x e^{-\lambda x}\right)\left(\lambda^{2} y e^{-\lambda y}\right) d y d x=\frac{3}{2 \lambda},
$$

\footnotetext{
${ }^{53}$ Such $v^{*}$ exists as $Y \geq{ }_{l r} X+V_{i}$ which implies that $\operatorname{Pr}\left(Y \geq X+V_{i}\right)=\int \operatorname{Pr}(Y \geq X+v) f(v) d v \geq 1 / 2$.

${ }^{54}$ Recall that the sum of two exponentially distributed random variables with parameter $\lambda$ has a distribution with density $f(x)=\lambda^{2} x e^{-\lambda x}$.
} 
whereas if firm $i$ deviates we have

$$
\pi\left(\sigma_{1}^{\prime \prime} ; \sigma^{\prime \prime}\right)+\pi\left(\sigma_{2}^{\prime \prime} ; \sigma^{\prime \prime}\right)=\int_{0}^{\infty} \int_{0}^{\infty}|x-y|\left(\lambda^{2} x e^{-\lambda x}\right)\left(\lambda e^{-\lambda y}\right) d y d x=\frac{3}{2 \lambda} .
$$

Therefore, the unilateral deviation to join $\sigma_{1}$ is not strictly profitable, which guarantees the stability of $\sigma .{ }^{55}$

(b) Suppose that $V \sim \Gamma(\alpha, 1)$ with $\alpha=1 / 4$. As before, Proposition 2 implies that, given the coalition profile $\sigma$, forming an individual coalition $\{i\}$ does not represent a profitable deviation for any firm $i$. Moreover, we have

$$
\begin{aligned}
\pi\left(\sigma_{1} ; \sigma\right)+\pi\left(\sigma_{2} ; \sigma\right) & =\int_{0}^{\infty} \int_{0}^{\infty}|x-y| \frac{e^{-x} x^{2 a-1}}{\Gamma(2 a)} \frac{e^{-y} y^{2 a-1}}{\Gamma(2 a)} d x d y=0.63662 \\
\pi\left(\sigma_{1}^{\prime \prime} ; \sigma^{\prime \prime}\right)+\pi\left(\sigma_{2}^{\prime \prime} ; \sigma^{\prime \prime}\right) & =\int_{0}^{\infty} \int_{0}^{\infty}|x-y| \frac{e^{-x} x^{2 a-1}}{\Gamma(2 a)} \frac{e^{-y} y^{a-1}}{\Gamma(a)} d x d y=0.545096, \quad \text { and } \\
\pi(\mathcal{N}) & =0.5
\end{aligned}
$$

Hence, the coalition profile $\sigma$ is uniquely stable, which concludes the proof of Corollary 3.

Proof of Lemma 2: In the limited-value case, consider a coalition profile $\sigma=\left(\sigma_{1}, . ., \sigma_{J}\right)$ with $n_{j} \leq \bar{n} \leq n_{k}$. Let $\left(W_{1}, W_{2}, \ldots, W_{J}\right)$ denote the associated coalitions' valuations. Firm $i$ 's move from coalition $\sigma_{j}$ to $\sigma_{k}$ results in another coalition profile $\sigma^{\prime}=\left(\sigma_{1}^{\prime}, \sigma_{2}^{\prime}, \ldots, \sigma_{J}^{\prime}\right)$ with $\sigma_{j}^{\prime}=\sigma_{j} \backslash\{i\}, \sigma_{k}^{\prime}=\sigma_{k} \cup\{i\}$, and $\sigma_{h}^{\prime}=\sigma_{h}$ for $h \neq j, k$.

It is without loss to assume that coalition $\sigma_{k}^{\prime}$ selects $\bar{n}$ existing members, but not firm $i$, to form the coalition valuation $W_{k}^{\prime}$. Then, coalition $\sigma_{k}^{\prime}$ 's valuation $W_{k}^{\prime}$ is identical to $W_{k}$. It follows that, for the coalition profile $\sigma^{\prime}$, the coalitions' valuations $\left(W_{1}^{\prime}, W_{2}^{\prime}, \ldots, W_{J}^{\prime}\right)$ are such that $W_{j}^{\prime}=W_{j}-V_{i}$, and $W_{h}^{\prime}=W_{h}$ for every $h \neq j$. Because every coalition bids their own valuation, the second highest bid under $\sigma^{\prime}$ is (weakly) lower than the second highest bid under $\sigma$ for every realization valuations, and strictly lower for some realizations in which $W_{j}$ is the second highest bid under $\sigma$ and $V_{i}>0$.

Proof of Proposition 3: (i) If $\bar{n}=N$, only the grand coalition can be stable; (ii) if $\bar{n}<N \leq 2 \bar{n}$, from Corollary 2 , it is immediate to see that $\sigma=\left\{\sigma_{1}, \sigma_{2}\right\}$ with $n_{1}=\bar{n}$, and $n_{2}=N-\bar{n}$ yields the maximum potential revenue for the seller. Therefore, $R^{*}(N, \bar{n}) \leq w_{(\widetilde{\sigma}, 2)}$, and the bound is attained if $\sigma$ is stable; (iii) if $N \geq 2 \bar{n}+1$, for any stable coalition profile

\footnotetext{
${ }^{55}$ Note tha the expected profit of the grand coalition is $\pi(\mathcal{N})=\frac{2}{\lambda}>\frac{3}{2 \lambda}$, which immediately guarantees the stability of the grand coalition without invoking Lemma 1.
} 
$\sigma^{\prime}=\left(\sigma_{1}^{\prime}, \ldots, \sigma_{J}^{\prime}\right)$, Corollary 2 implies that $n_{1} \geq \cdots \geq n_{J-1} \geq \bar{n}$. The total value of each coalition $\sigma_{j}^{\prime}$ is $W_{j}^{\prime} \equiv \sum_{i=1}^{\min \left\{n_{j} \bar{n}\right\}} V_{i}$. Let $W_{j}^{\prime} \equiv 0$ for $j=J+1, J+2, \ldots, K$. Similarly, let $W_{k}$ denote the value of each coalition of $\sigma_{k}$ in the coalition profile $\widetilde{\sigma}$ defined in the claim. Then, for every $k=1,2, \ldots, K$, we have $W_{k}^{\prime} \leq_{l r} W_{k}$, which implies $W_{k}^{\prime} \leq_{s t} W_{k}$. Observe that a function that finds the second highest number from a vector of integers is increasing in each argument. It follows from Theorem $\mathrm{A} 3$ that $W_{\left(\sigma^{\prime}, 2\right)} \leq_{s t} W_{(\widetilde{\sigma}, 2)}$. Therefore, $R^{*}(N, \bar{n}) \leq w_{(\widetilde{\sigma}, 2)}$, and the bound is attained if $\widetilde{\sigma}$ is stable

Proof of Corollary 4: Points (b) and (c) of Corollary 2 imply that in the presence of a ceiling the coalition profile $\widetilde{\sigma}$ is the only remaining candidate to be stable. For it to be stable, we need to guarantee that (i) if $n_{K}<\bar{n}$, no firm belonging to a coalition of size $\bar{n}$ has a profitable deviation in joining $\sigma_{K}$; (ii) if $n_{K}<\bar{n}$, no firm belonging to $\sigma_{K}$ has a profitable deviation in participating in the auction as individual bidder, and (iii) no firm belonging to a coalition of size $\bar{n}$ has a profitable deviation in participating in the auction as individual bidder. However, in the proof of Proposition 2, we defined

$$
X \equiv \sum_{a \in \sigma_{j} \backslash\{i\}} V_{a}, \quad Y \equiv \sum_{a \in \sigma_{k}} V_{a}, \quad Z \equiv \max _{h \neq j, k} \sum_{a \in \sigma_{h}} V_{a},
$$

and for any $x, y, v_{i}, z \geq 0$,

$$
\phi\left(x, y ; v_{i}, z\right) \equiv \max \left\{x+v_{i}, y, z\right\}-\max \left\{\min \left\{x+v_{i}, y\right\}, z\right\} .
$$

We showed that

$$
\mathbf{E}\left[\phi\left(X, Y ; V_{i}, Z\right)\right]<\mathbf{E}\left[\phi\left(Y, X ; V_{i}, Z\right)\right] .
$$

This result holds for any $n_{j}>0$,and in particular for $n_{j}=1$ (in this case, note that $X=0$ ). The result guarantees that (i), (ii), and (iii) do not represent profitable deviations in the coalition profile $\widetilde{\sigma}$. Therefore, in the presence of a coalitions' size ceiling equal to $\bar{n}$, the coalition profile $\widetilde{\sigma}$ is uniquely stable and the upper bound for the seller's expected revenue $w_{(\widetilde{\sigma}, 2)}$ is uniquely obtained.

Proof of Proposition 4: Let $\sigma=\left(\sigma_{1}, \sigma_{2}, \ldots\right)$ be a coalition profile such that $n_{1} \geq n_{2} \geq 1$. For any $i \in \sigma_{2}$, let $\sigma^{\prime}=\left(\sigma_{1}^{\prime}, \sigma_{2}^{\prime}, \ldots\right)$ be such that $\sigma_{1}^{\prime}=\sigma_{1} \cup\{i\}, \sigma_{2}^{\prime}=\sigma_{2} \backslash\{i\}$, and $\sigma_{k}^{\prime}=\sigma_{k}$ for $k \neq 1,2$. Then,

$$
\pi\left(\sigma_{1} ; \sigma\right)+\pi\left(\sigma_{2} ; \sigma\right)<\pi\left(\sigma_{1}^{\prime} ; \sigma^{\prime}\right)+\pi\left(\sigma_{2}^{\prime} ; \sigma^{\prime}\right)
$$


is sufficient to show that $\sigma=\{\mathcal{N}\}$ is the only stable coalition profile. To show (10), we apply a modified proof of Theorem A1 (see Section 6.1). Define

$$
X \equiv \max \left\{V_{j} \mid j \in \sigma_{2} \backslash\{i\}\right\}, \quad Y \equiv \max \left\{V_{k} \mid k \in \sigma_{1}\right\}, \quad \text { and } \quad Z \equiv \max \left\{V_{k} \mid k \notin \sigma_{1} \cup \sigma_{2}\right\}
$$

Let $X^{\prime} \equiv \max \left\{X, V_{i}\right\}$ and $Y^{\prime} \equiv \max \left\{Y, V_{i}\right\}$. Also, for any $x, y, v_{i}, z \geq 0$, define $x^{\prime} \equiv \max \left\{x, v_{i}\right\}$ and

$$
\phi\left(x, y ; v_{i}, z\right) \equiv \max \left\{x^{\prime}, y, z\right\}-\max \left\{\min \left\{x^{\prime}, y\right\}, z\right\}
$$

Then,

$$
\begin{aligned}
& \pi\left(\sigma_{1} ; \sigma\right)+\pi\left(\sigma_{2} ; \sigma\right)=\mathbf{E}\left[\phi\left(X, Y ; V_{i}, Z\right)\right], \text { and } \\
& \pi\left(\sigma_{1}^{\prime} ; \sigma^{\prime}\right)+\pi\left(\sigma_{2}^{\prime} ; \sigma^{\prime}\right)=\mathbf{E}\left[\phi\left(Y, X ; V_{i}, Z\right)\right] .
\end{aligned}
$$

Note that, for any $V_{i}=v_{i}$ and $Z=z$, we have

$$
\begin{aligned}
& \phi\left(x, y ; v_{i}, z\right)=\max \left\{x^{\prime}, y, z\right\}-\max \left\{\min \left\{x^{\prime}, y\right\}, z\right\}, \\
& \phi\left(y, x ; v_{i}, z\right)=\max \left\{y^{\prime}, x, z\right\}-\max \left\{\min \left\{y^{\prime}, x\right\}, z\right\}
\end{aligned}
$$

and whenever $x \leq y$, we have

$$
\phi\left(y, x ; v_{i}, z\right)-\phi\left(x, y ; v_{i}, z\right)=\max \left\{\min \left\{x^{\prime}, y\right\}, z\right\}-\max \{x, z\} \geq 0
$$

If $X=0$ (i.e., $n_{2}=1$ ), then

$$
\phi\left(y, 0 ; v_{i}, z\right)-\phi\left(0, y ; v_{i}, z\right)=\max \left\{\min \left\{v_{i}, y\right\}, z\right\}-z \geq 0
$$

with a strict inequality for some $y, v_{i}, z$. In this case, (10) follows from the continuity of $\phi$.

Suppose instead that $X \neq 0$ (i.e., $n_{2}>1$ ). Note that the density functions of $X$ and $Y$ are

$$
\begin{aligned}
& F_{X}(x) \equiv \operatorname{Pr}\left(V_{\left(n_{2}-1,1\right)} \leq x\right)=F^{n_{2}-1}(x) \quad \Longrightarrow f_{X}(x)=\left(n_{2}-1\right) F^{n_{2}-2}(x) f(x), \\
& F_{Y}(y) \equiv \operatorname{Pr}\left(V_{\left(n_{1}, 1\right)} \leq y\right)=F^{n_{1}}(y) \quad \Longrightarrow f_{Y}(y)=n_{1} F^{n_{1}-1}(y) f(y) .
\end{aligned}
$$

The likelihood ratio $\frac{f_{Y}(x)}{f_{X}(x)}=\frac{n_{1}}{n_{2}-1} F^{n_{1}-n_{2}+1}(x)$ is strictly increasing in $x \in \operatorname{supp}(F)$, i.e.,

$$
f_{X}(x) f_{Y}(y)>f_{X}(y) f_{Y}(x), \quad \text { for all } x<y
$$


Note that, for any $V_{i}=v_{i}$ and $Z=z$,

$$
\begin{aligned}
& \mathbf{E}_{X, Y}\left[\phi\left(Y, X ; v_{i}, z\right)\right]-\mathbf{E}_{X, Y}\left[\phi\left(X, Y ; v_{i}, z\right)\right] \\
= & \int_{y} \int_{y \geq x}\left(\phi\left(y, x ; v_{i}, z\right)-\phi\left(x, y ; v_{i}, z\right)\right) f_{X}(x) f_{Y}(y) d x d y \\
& +\int_{y} \int_{y \leq x}\left(\phi\left(y, x ; v_{i}, z\right)-\phi\left(x, y ; v_{i}, z\right)\right) f_{X}(x) f_{Y}(y) d x d y .
\end{aligned}
$$

We can rewrite the last term as

$$
\begin{aligned}
& \int_{y} \int_{y \leq x}\left(\phi\left(y, x ; v_{i}, z\right)-\phi\left(x, y ; v_{i}, z\right)\right) f_{X}(x) f_{Y}(y) d x d y \\
& =\int_{x} \int_{y \leq x}\left(\phi\left(y, x ; v_{i}, z\right)-\phi\left(x, y ; v_{i}, z\right)\right) f_{X}(x) f_{Y}(y) d y d x \\
& =\int_{y} \int_{x \leq y}\left(\phi\left(x, y ; v_{i}, z\right)-\phi\left(y, x ; v_{i}, z\right)\right) f_{X}(y) f_{Y}(x) d x d y .
\end{aligned}
$$

Then,

$$
\begin{aligned}
& \mathbf{E}_{X, Y}\left[\phi\left(Y, X ; v_{i}, z\right)\right]-\mathbf{E}_{X, Y}\left[\phi\left(X, Y ; v_{i}, z\right)\right] \\
= & \int_{y} \int_{y \geq x}\left(\phi\left(y, x ; v_{i}, z\right)-\phi\left(x, y ; v_{i}, z\right)\right)\left(f_{X}(x) f_{Y}(y)-f_{X}(y) f_{Y}(x)\right) d x d y \geq 0 .
\end{aligned}
$$

For some $x, y, v_{i}$, and $z$, the inequality (11) holds strictly. Because $\phi$ is continuous, (12) holds strictly in a neighborhood of $\left(v_{i}, z\right)$, which guarantees (10) to hold. 


\section{$6 \quad$ References}

Albano G. "Competition in Public Procurement Markets." OECD Working Party No. 2 on Competition and Regulation, Background Paper, 19 June 2017.

Albano G., Spagnolo, G. and Zanza, M. "Regulating Joint Bidding in Public Procurement." Journal of Competition Law \& Economics, Vol. 5 (2008), pp. 335-360.

Agranov, M. and Yariv, L. "Collusion through Communication in Auctions." Games and Economic Behavior, Vol. 107 (2018), pp. 93-108.

Asker, J. "A Study of the Internal Organization of a Bidding Cartel." American Economic Review, Vol. 100 (2010), pp. 724-762.

Avery C., and Hendershott, T. "Bundling and Optimal Auctions of Multiple Products." The Review of Economic Studies, Vol. 67 (2000), pp. 483-497.

Baccara M., and Yariv, L. "Homophily in Peer Groups", American Economic Journal: Microeconomics, Vol. 5 (2013), pp. 69-96.

Baccara, M., and Yariv, L. "Choosing Peers: Homophily and Polarization in Groups." Journal of Economic Theory, Vol. 165 (2016), pp. 152-178.

Baik, K.H., Kim, I., and Na, S. "Bidding for a Group-Specific Public-Good Prize." Journal of Public Economics, Vol. 82 (2001), pp. 415-429.

Bernheim, D., and Whinston, M. "Multimarket Contact and Collusive Behavior." RAND Journal of Economics, Vol. 21 (1990), pp. 1-26.

Brickley, P. (2011), "Nortel \$4.5-Billion Patent Sale to Apple, Microsoft, Others Approved." Wall Street Journal, 7/11/2011.

Brusco, S., and Lopomo, G. "Collusion via Signalling in Simultaneous Ascending Bid Auctions with Heterogeneous Objects with and without Complementarities." Review of Economic Studies, Vol. 69 (2002), pp. 407-436.

Buchanan, J.M. "An Economic Theory of Clubs." Economica, Vol. 32 (1965), pp. 1-14.

Bulow, J., and Roberts, J. "The Simple Economics of Optimal Auctions." The Journal of Political Economy, Vol. 97 (1989), pp. 1060-1090. 
Cantillon, E. "The Effect of Bidders' Asymmetries on Expected Revenue in Auctions." Games and Economic Behavior, Vol. 62 (2008), pp. 1-25.

Chassang, S., and Ortner, J. "Collusion in Auctions with Constrained Bids: Theory and Evidence from Public Procurement." Journal of Political Economy, Vol. 127 (2019), pp. 2269-2300.

Chatterjee, K., Mitra, M., and Mukherjee, C. "Bidding Rings: A Bargaining Approach." Games and Economics Behavior, Vol. 103 (2017), pp. 67-82.

Che, Y.-K., and Kim, J. "Robustly Collusion-Proof Implementation." Econometrica, Vol. 74 (2006), pp. 1063-1107.

Che, Y.-K., and Kim, J. "Optimal Collusion-Proof Auctions." Journal of Economic Theory, Vol. 144 (2009), pp. 565-603.

Che, Y.-K., Condorelli, D., and Kim, J. "Weak Cartels and Collusion-Proof Auctions." Journal of Economic Theory, Vol. 178 (2018), pp. 398-435.

Chen, C.-L., and Tauman, Y. "Collusion in one-shot second-price auctions." Economic Theory, Vol. 28 (2006), pp. 145-172.

Cho, I.-K., Jewell, K., and Vohra, R. "A Simple Model of Coalitional Bidding." Economic Theory, Vol. 19 (2002), pp. 435-457.

Deb, R., and Razzolini, L. "Auction-Like Mechanisms for Pricing Excludable Public Goods." Journal of Economic Theory, Vol. 88 (2001), pp. 340-368.

Decarolis, F., Goldmanis, M. and Penta, A. "Marketing Agencies and Collusive Bidding in Online Ad Auctions." Management Science. Vol. 66 (2020), pp. 4359-4919.

Decarolis, F., and Rovigatti, G. "From Mad Men to Maths Men: Concentration and Buyer Power in Online Advertising." CEPR Discussion Paper 13897, 2020.

Demange, G. "Intermediate Preferences and Stable Coalition Structures." Journal of Mathematical Economics, Vol. 23 (1994), pp. 45-58.

Dutta, B., and Vohra, R. "Incomplete Information, Credibility and the Core." Mathematical Social Sciences, Vol. 50 (2005), pp.148 - 165.

Ellickson, B., Grodal, B., Scotchmer, S., and Zame, W. R. "Clubs and the Market." Econometrica, Vol. 67 (1999), pp. 1185-1218. 
Ernst \& Young Inc. (2011), Seventy-First Report of the Monitor In the Matter of the Companies' Creditors Arrangement Action, R.S.C. 1985, c. C-36, As Amended and the Matter of a Plan of Compromise or Arrangement of Nortel Networks Corporation, et al, Ontario Superior Court of Justice (Commercial List), 7/6/2011, Court File No. 09-CL-7950.

Eso, P., and Schummer, J. "Bribing and Signaling in Second Price Auctions." Games and Economic Behavior, Vol. 47 (2004), pp. 299-324.

Fang H., and Norman, P. "Optimal Provision of Multiple Excludable Public Goods." American Economic Journals: Microeconomics, Vol. 2 (2010), pp. 1-37.

Garratt, R., Tröger, T., and Zheng, C. "Collusion via Resale." Econometrica, Vol. 77 (2009), pp. 1095-1136.

Graham, D. and Marshall, R. "Collusive Bidder Behavior at Single-Object Second-Price and English Auctions." Journal of Political Economy, Vol. 95 (1987), pp. 1217-1239.

Harris, M., and Raviv, A. "Allocation Mechanisms and the Design of Auctions." Econometrica, Vol. 49 (1981), pp. 1477-1499.

Hoppe, H.C., Jehiel, P., and Moldovanu, B. "Licence Auctions and Market Structure." Journal of Economics and Management Strategy, Vol. 15 (2006), pp. 371-396.

Jehiel, P., and Moldovanu, B. "Strategic Non-Participation." The RAND Journal of Economics, Vol. 27 (1996), pp. 84-98.

Jehiel, P., Moldovanu, B., and Stacchetti, E. "How (not) to Sell a Nuclear Weapon." The American Economic Review, Vol. 86 (1996), pp. 814-829.

Katz, M. L., and Shapiro, C. "On the Licensing of Innovation." The RAND Journal of Economics, Vol. 16 (1985), pp. 504-520.

Kemperer, P. Auctions: Theory and Practice. Princeton: Princeton University Press, 2004.

Kirkegaard, R. "Asymmetric First Price Auctions." Journal of Economic Theory, Vol. 144 (2009), pp.1617-1635.

Kirkegaard, R. "A Mechanism Design Approach to Ranking Asymmetric Auctions." Econometrica, Vol. 80 (2012), pp. 2349-2364.

Kivetz, G. and Tauman Y. "Simple collusive agreements in one-shot first-price auctions." Games and Economic Behavior, Vol. 69 (2010), pp. 138-149. 
Laffont, J.-J., and Martimort, D. "Collusion Under Asymmetric Information." Econometrica, Vol. 65 (1997), pp. 875-911.

Laffont, J.-J., and Martimort, D. "Mechanism Design with Collusion and Correlation." Econometrica, Vol. 68 (2000), pp. 309-342.

Loertscher, S., and Marx, L. "Club Good Intermediaries." International Journal of Industrial Organization, Vol. 50 (2017), pp. 430-459.

Lopomo, G., Marshall, R., and Marx, L. "Inefficiency of Collusion at English Auctions." Contributions to Theoretical Economics, Vol 5 (2005), Article 1.

Marshall, R., and Marx, L. The Economics of Collusion: Cartels and Bidding Rings. Cambridge: MIT Press, 2012.

Maskin, E., and Riley, J. "Optimal Multi-Unit Auctions." In F. Hahn, ed., The Economics of Missing Markets, Information, and Games. Oxford: Oxford University Press, 1989.

Maskin E., and Riley, J. G. "Asymmetric Auctions." Review of Economic Studies, Vol. 67 (2000a), pp. 413-438.

Maskin, E., and Riley, J.G. "Equilibrium in Sealed High Bid Auctions." Review of Economic Studies, Vol. 67 (2000b), pp. 439-454.

McAfee, R. P., and McMillan, J. "Bidding Rings." The American Economic Review, Vol. 82 (1992), pp. 579-599.

Milgrom, P. Putting Auction Theory to Work. Cambridge: Cambridge University Press, 2004.

Norman, P. "Efficient Mechanism for Public Goods with Use Exclusions." Review of Economic Studies, Vol. 71 (2004), pp. 1163-1188.

Pavlov, G. "Auction design in the presence of collusion." Theoretical Economics, Vol. 3 (2008), pp. 383-429.

Porter, R. "Book Review: Anatomy of an Auction: Rare Books at Ruxley Lodge, 1919." Journal of Political Economy, Vol. 100 (1992), pp. 433-436.

Shaked, M., and Shanthikunar, J.G. Stochastic Orders, New York: Springer-Verlag, 2007.

Rachmilevitch, S. "Bribing in First-Price Auctions." Games and Economic Behavior, Vol. 77 (2013), pp. 214-228. 
Ray, D. and Vohra, R. "A theory of endogenous coalition structures." Games and Economic Behavior, Vol. 26 (1999), pp. 286-336.

Ray D., and Vohra, R. "Coalition Formation." In H. P.Young and S. Zamir, eds., Handbook of Game Theory Vol. 4. Amsterdam: North Holland Elsevier, 2015.

Richardson K., Oliver, , E., and Costa, M. "The 2017 brokered patent market - the fightback begins." IAM Magazine Vol. 87 (2018), pp. 8-22.

Troyan, P. "Collusion and signaling in auctions with interdependent values." Journal of Economic Theory, Vol. 170 (2017), pp. 319-345.

Weber R. "Multi-Object Auctions." In R. Engelbrecht-Wiggans, R.M. Stark, and M. Shubik, eds., Competitive Bidding, Auctions, and Procurement. New York: New York University Press, 1983. Reprinted in P. Klemperer, ed., The Economic Theory of Auctions II. Cheltenham: Edward Elgar Publishing Ltd., 2000.

Wooders, M., Cartwright, E. and Selten, R. "Behavioral Conformity in Games with Many Players." Games and Economic Behavior, Vol. 57 (2006), pp. 347-360.

Zheng, C. "Bidding Collusion without Passive Updating." Journal of Mathematical Economics, Vol. 85 (2019a), pp. 70-77.

Zheng, C. "Necessary and Sufficient Conditions for Peace: Implementability versus Security." Journal of Economic Theory, Vol. 180 (2019b), pp. 135-166. 


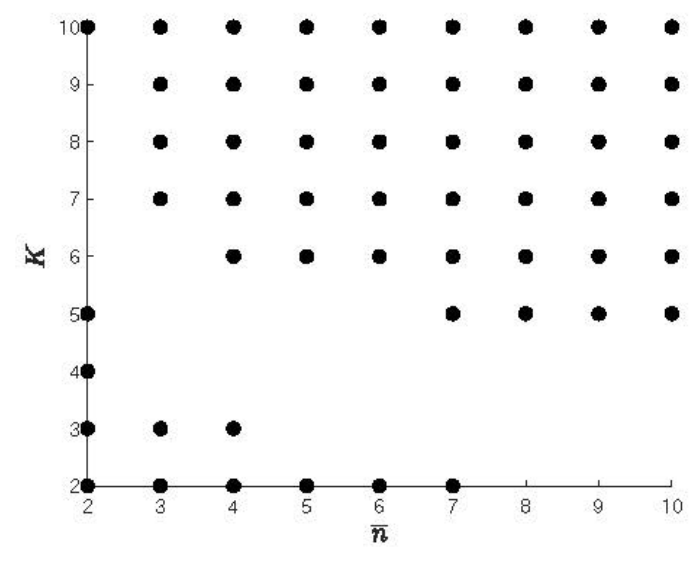

(a) $\alpha=0.3$

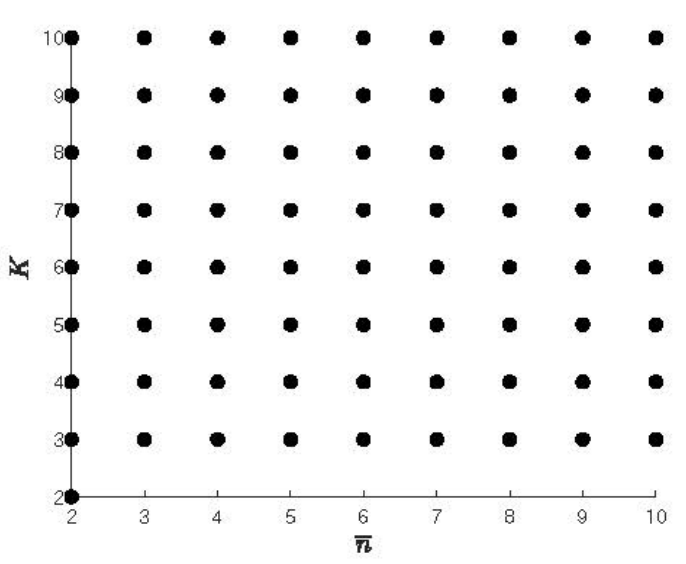

(c) $\alpha=3$

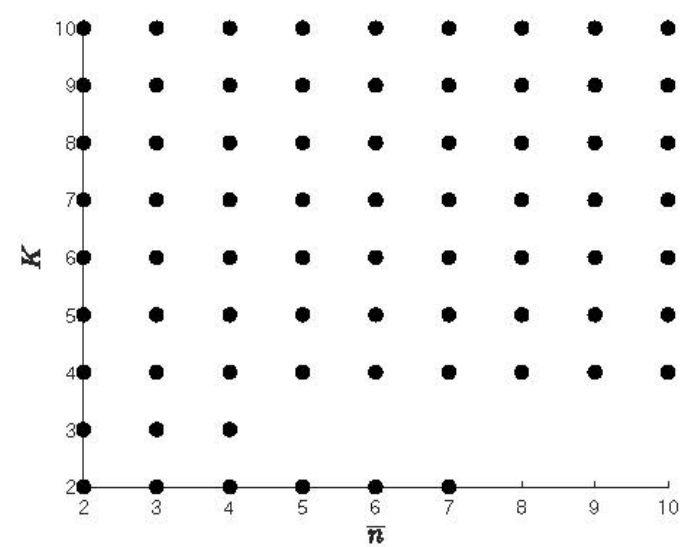

(b) $\alpha=0.5$

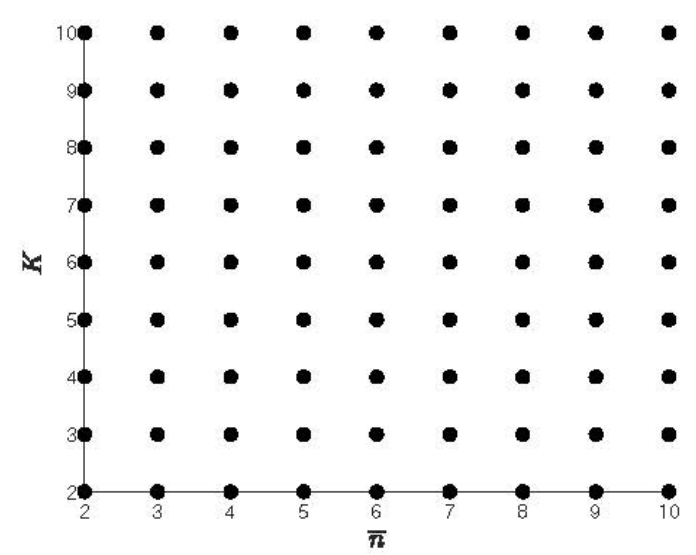

(d) $\alpha=7$

Figure 1: Relative Performance of Patent and Multi-License Auctions for $V_{i} \sim \operatorname{Gamma}(\alpha, \beta)$. 